\title{
Effects of radial radio-frequency field inhomogeneity on MAS solid-state NMR experiments
}

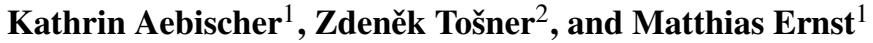 \\ ${ }^{1}$ Physical Chemistry, ETH Zürich, Vladimir-Prelog-Weg 2, 8093 Zurich, Switzerland \\ ${ }^{2}$ Department of Chemistry, Faculty of Science, Charles University, Hlavova 8, 12842 Prague 2, Czech Republic
}

Correspondence: Matthias Ernst (maer@ethz.ch)

Received: 6 May 2021 - Discussion started: 7 May 2021

Revised: 7 June 2021 - Accepted: 14 June 2021 - Published: 1 July 2021

\begin{abstract}
Radio-frequency field inhomogeneity is one of the most common imperfections in NMR experiments. They can lead to imperfect flip angles of applied radio-frequency (rf) pulses or to a mismatch of resonance conditions, resulting in artefacts or degraded performance of experiments. In solid-state NMR under magic angle spinning (MAS), the radial component becomes time-dependent because the rf irradiation amplitude and phase is modulated with integer multiples of the spinning frequency. We analyse the influence of such time-dependent MAS-modulated rf fields on the performance of some commonly used building blocks of solid-state NMR experiments. This analysis is based on analytical Floquet calculations and numerical simulations, taking into account the time dependence of the rf field. We find that, compared to the static part of the rf field inhomogeneity, such time-dependent modulations play a very minor role in the performance degradation of the investigated typical solid-state NMR experiments.
\end{abstract}

\section{Introduction}

Radio-frequency (rf) field inhomogeneity describes the spatial inhomogeneity of the rf field inside the coil or sample volume and is one of the major experimental imperfections that leads to artefacts or reduced efficiency in NMR experiments. The magnitude of the rf field amplitude distribution over the sample space can be estimated with a nutation experiment (Torrey, 1949; Barnaal and Lowe, 1963). Measuring such nutation spectra of thin sample slices placed along the rotor axis allows the characterization of the spatial rf field distribution along the coil axis (Nishimura et al., 2001; Paulson et al., 2004). The full spatial distribution, however, is only accessible using gradient methods (Guenneugues et al., 1999; Odedra and Wimperis, 2013) that are typically not available in solid-state NMR probes. Alternative approaches include the measurement of the rf field amplitude using the ball shift experiment (Maier and Slater, 1952), numerical simulations based on finite elements, or approximative analytical solutions of the Maxwell equations (Engelke, 2002; Tošner et al., 2017, 2018). The design of the coil geometry has a major influence on the magnitude and distribution of the rf field amplitude over the active sample volume, and different geometries have been proposed to improve the rf homogeneity (Idziak and Haeberlen, 1982; Privalov et al., 1996; Li et al., 2006). However, in solid-state NMR probes, solenoid coils along the sample spinning axis are most commonly used due to the high, achievable rf field amplitudes. The gap between the rotor and the coil is minimized in order to optimize the filling factor. This design choice typically leads to large rf field inhomogeneity that can manifest itself in reduced efficiency in experiments such as cross-polarization (Hartmann and Hahn, 1962; Stejskal et al., 1977), homonuclear decoupling (Bielecki et al., 1989, 1990; Mote et al., 2016), heteronuclear decoupling (Purusottam et al., 2015; Frantsuzov et al., 2017), symmetry-based recoupling sequences (Levitt, 2007), or even pulsed recoupling experiments like rotational-echo double-resonance (REDOR; Nishimura et al., 2001).

Reducing the magnitude of the rf field inhomogeneity can be achieved experimentally by physically restricting the sample along the rotor axis or even to a sphere in the centre of the rotor (Lindon et al., 2009). Alternatively, gradients can be 
used for a virtual sample restriction (Charmont et al., 2000), but since gradients are not very common in solid-state NMR probes, this approach is rarely used. Another possibility is radio or nutation-frequency-selective pulses that can be used for the same purpose (Charmont et al., 2002; Aebischer et al., 2020). All these methods, however, are accompanied by a reduction in signal due to the restriction of the measured sample volume to a smaller part of the coil volume.

In solid-state NMR under magic angle spinning (MAS) conditions, the radial component of the rf field is modulated by time (Levitt et al., 1988; Tekely and Goldman, 2001; Goldman and Tekely, 2001; Tošner et al., 2017), leading to further potential complications in the experiments. Such MAS-induced time-dependent radio-frequency fields could give rise to additional or modified resonance conditions or to other changes in the effective Hamiltonian generated by the pulse sequence. The importance of such time-dependent terms was first described in rotary resonance recoupling (Levitt et al., 1988), where it leads to changes in the observed line shape. Besides the appearance of additional sidebands in cross-polarization experiments (Tekely and Goldman, 2001; Goldman and Tekely, 2001), nutation spectra (Elbayed et al., 2005), and reported phase distortions and loss of magnetization in MLEV-16 sequences under MAS (Piotto et al., 2001), there have been very few studies of the effects of such modulations of the amplitude and phase of the rf field caused by MAS rotation. These modulations have been included in the design of a heteronuclear polarization transfer scheme based on optimal control strategies (Tošner et al., 2018), where impressive gains have been shown. These improvements prompted us to investigate potential effects of such MAS-modulated radio-frequency field amplitudes and phases on basic building blocks in common solid-state NMR pulse sequences in more detail. The approach we have chosen is rather simple. We use analytical approaches based on Floquet theory (Leskes et al., 2010; Scholz et al., 2010) and numerical simulations based on computed rf field distributions in a typical MAS rotor to characterize the time evolution of the density operator under MAS rotation with and without time-dependent rf field amplitudes and phases. The computational approach allows us to investigate the time evolution of the density operator in different spatial parts of the rotor and to magnify the amplitude or phase modulations of the rf field to obtain a better picture of their importance.

\section{Radio-frequency fields in solenoid coils}

To illustrate the magnitude and distribution of the rf field amplitude and phase over the active sample volume in some typical MAS NMR probes, rf field distributions were calculated based on Engelke (2002) and Tošner et al. (2017). Figure 1 shows the relative amplitude and phase of the rf field in a cylindrical coordinate system as a function of $z$ (the axis along the rotor axis) and $r$ (the radial direction) for three common probe designs with MAS rotors of 3.2, 1.9, and $1.3 \mathrm{~mm}$ outer diameter. In these plots, the angle $\vartheta=90^{\circ}$ was chosen. The maximum intensity of the rf amplitude distribution was used as the reference point for the relative amplitude $\omega_{\text {rel }}(\boldsymbol{r})$; hence, a value of 1 means that the amplitude experienced at this position corresponds to the nominal rf amplitude. The rf phase was computed relative to the centre point of the rotor with $(r, z)=(0,0)$. One can clearly see the decay in the rf amplitude towards the edges of the rotor along the rotor axis (large $z$ values), while phase errors mostly occur for large $r$ and $z$ values.

The radial dependence of the relative rf field amplitude and phase as a function of the angle $\vartheta$ is shown in Fig. 2 for different values of $z$ and $r$ for the $3.2 \mathrm{~mm}$ MAS probe. Under sample rotation, the angle $\vartheta$ varies as a function of time, and the rf field amplitude and phase are periodically modulated with the rotor frequency. The trajectories in Fig. 2 clearly show that the magnitude of these amplitude and phase modulations increases towards the edges of the rotor. We, therefore, expect crystallites located at large $r$ and $z$ values to experience the strongest modulations of the rf field amplitude and phase.

For numerical simulations of spin dynamics, the numerical values for $\omega_{1, \text { rel }}(\boldsymbol{r})$ and $\phi_{\text {rel }}(\boldsymbol{r})$, obtained from simulations of the rf field distribution shown in Figs. 1 and 2, were used directly as input. For analytical calculations based on Floquet theory, a parameterization of the values using a Fourier series with the MAS frequency, $\omega_{\mathrm{r}}$, is more convenient and was obtained by fitting the following expressions:

$$
\begin{aligned}
& \omega_{\text {rel }}(t)=A_{0}^{(\mathrm{A})}+\sum_{n=1}^{\infty} A_{n}^{(\mathrm{A})} \cdot \cos \left(n \omega_{\mathrm{r}} t+\phi_{n}^{(\mathrm{A})}\right), \\
& \phi_{\text {rel }}(t)=A_{0}^{(\mathrm{P})}+\sum_{n=1}^{\infty} A_{n}^{(\mathrm{P})} \cdot \cos \left(n \omega_{\mathrm{r}} t+\phi_{n}^{(\mathrm{P})}\right)
\end{aligned}
$$

to the amplitude and phase changes of the rf field (see Fig. 2). Typically, terms of the Fourier series up to $n=4$ were used in the fits to characterize the time-dependent amplitude $\omega_{\text {rel }}(t)$ and phase $\phi_{\text {rel }}(t)$.

\section{Theory}

\subsection{Floquet description}

In the high field approximation, the total Hamiltonian in the rotating frame under MAS for a homonuclear spin system comprised of $N I$ spins is given by the following:

$$
\begin{aligned}
\hat{\mathcal{H}}(t)= & \sum_{n=-2}^{2} \sum_{p=1}^{N} \omega_{p}^{(n)} e^{i n \omega_{\mathrm{r}} \mathrm{t}} \hat{\boldsymbol{I}}_{z}+\sum_{p<q} \sum_{\substack{n=-2 \\
n \neq 0}}^{2} \omega_{p q}^{(n)} e^{i n \omega_{\mathrm{r}} t} \\
& \times\left[3 \hat{\boldsymbol{I}}_{p z} \hat{\boldsymbol{I}}_{q z}-\hat{\boldsymbol{I}}_{p} \cdot \hat{\boldsymbol{I}}_{q}\right]+\sum_{p<q} \omega_{p q}^{(0)} \hat{\boldsymbol{I}}_{p} \cdot \hat{\boldsymbol{I}}_{q}+\hat{\mathcal{H}}_{\mathrm{rf}}(t) .
\end{aligned}
$$



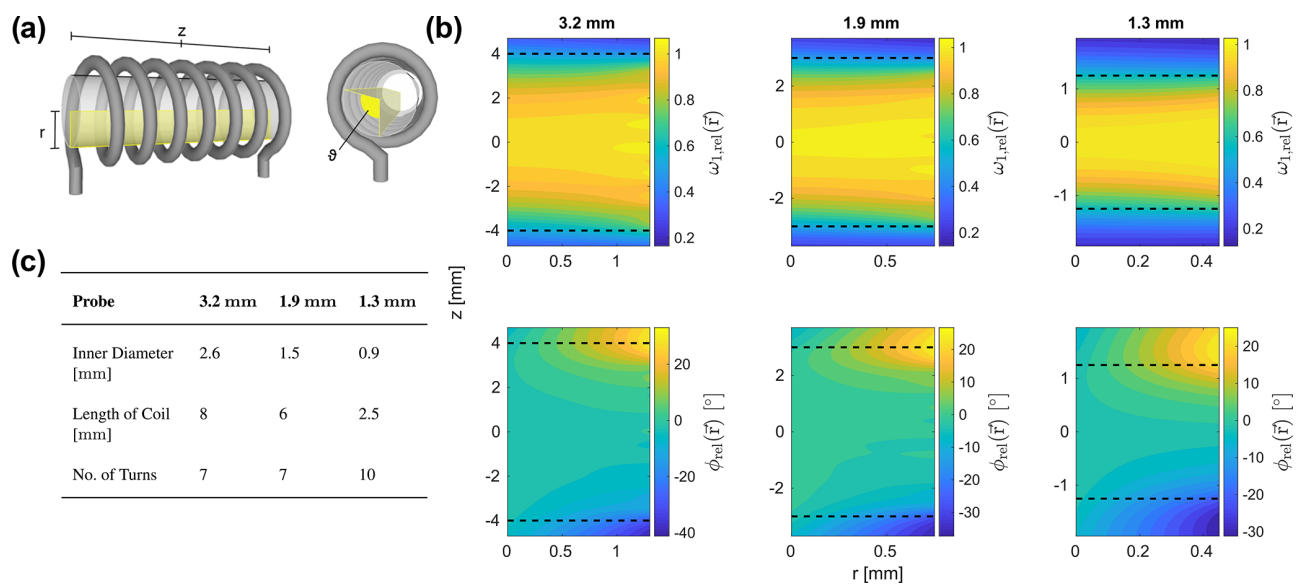

Figure 1. (a) Coil geometry of a typical $3.2 \mathrm{~mm}$ Bruker MAS probe. The position within the sample space is indicated by the cylindrical coordinates $r, z$, and $\vartheta$. (b) Spatial rf field distributions for typical probe designs with MAS rotors of 3.2, 1.9, and 1.3 mm outer diameter at a frequency of $600 \mathrm{MHz}$. Relative rf amplitudes $\omega_{\text {rel }}$ and phases $\phi_{\text {rel }}$ are shown as a function of the position within the active sample volume for $\vartheta=90^{\circ}$. The length and position of the solenoid coil is indicated by dashed lines. (c) Parameters of coil geometries of the 3.2, 1.9, and $1.3 \mathrm{~mm}$ MAS probes considered in this work. The $z$ values were sampled in steps of $0.05 \mathrm{~mm}$ for all three probes. The $r$ values were sampled in steps of $0.05 \mathrm{~mm}$ for the 3.2 and the $1.9 \mathrm{~mm}$ probes and in steps of $0.025 \mathrm{~mm}$ for the $1.3 \mathrm{~mm}$ probe. The sample volume considered in the numerical simulations and Floquet analyses presented in Sect. 5 was restricted to the length of the coil.
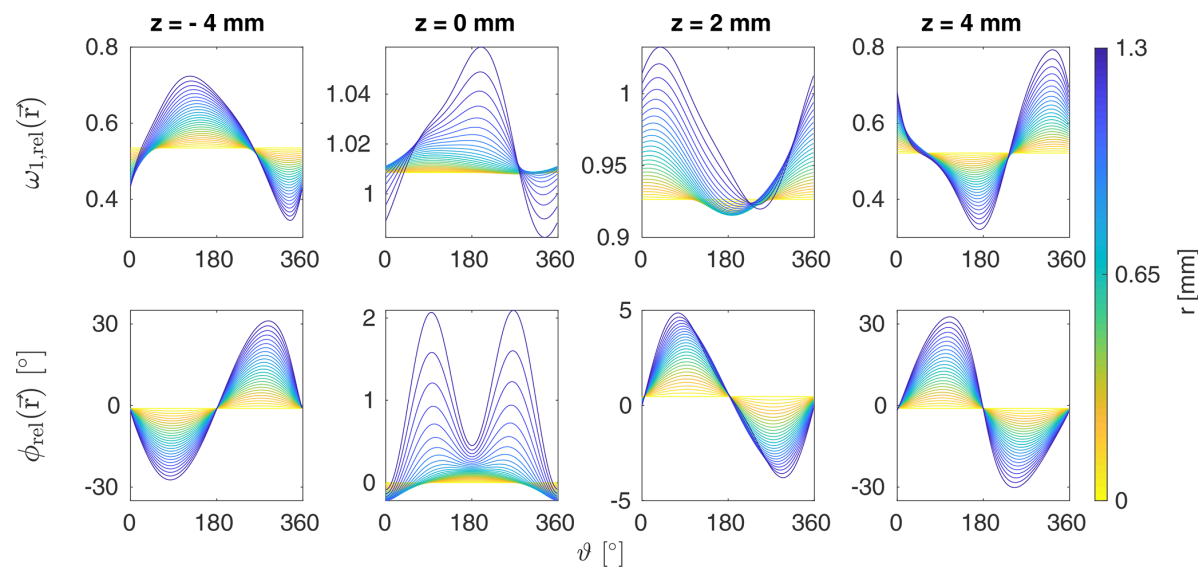

Figure 2. Relative rf amplitude $\omega_{1 \text {,rel }}$ and phase $\phi_{\text {rel }}$ as a function of $\vartheta$ for $z=-4,0,2$, and $4 \mathrm{~mm}$ and all $r$ values in the $3.2 \mathrm{~mm}$ MAS probe at a resonance frequency of $600 \mathrm{MHz}$. Under MAS, the rf amplitude and phase experienced by a crystallite will vary periodically with the rotor frequency. These time-dependent modulations of the rf field are strongest at the edges of the rotor for large $r$ and $z$ values.

Fourier components of the spatial tensors $\omega^{(n)}$ of the chemical shift of a spin $I_{p}$ and couplings between two spins $I_{p}$ and $I_{q}$ are given by the following:

$\omega_{p}^{(0)}=\Omega_{p}$

$\omega_{p}^{(n)}=\frac{2}{\sqrt{6}} d_{n, 0}^{2}\left(\theta_{\mathrm{m}}\right) e^{-i n \gamma} \sum_{m=-2}^{2} d_{m, n}^{2}(\beta) e^{-i m \alpha} \rho_{2, m}^{(p)}$

$\omega_{p q}^{(0)}=2 \pi J_{p q}$

$\omega_{p q}^{(n)}=\frac{1}{\sqrt{6}} d_{n, 0}^{2}\left(\theta_{\mathrm{m}}\right) e^{-i n \gamma} d_{0, n}^{2}(\beta) \rho_{2,0}^{(p q)}$

for the isotropic and anisotropic chemical shifts, the scalar $J$, and the anisotropic dipolar coupling. The sets of Euler angles $(\alpha, \beta, \gamma)$ describe the orientation of the tensors in the rotorfixed frame, and $d_{m, m^{\prime}}^{\ell}(\beta)$ denote the reduced Wigner matrix elements. The elements of the tensor in its principal axis system are denoted by $\rho_{\ell, m}$. The most general rf Hamiltonian for Eq. (3) is given by the following:

$\hat{\mathcal{H}}_{\mathrm{rf}}(t)=\omega_{1}(t) \sum_{p=1}^{N}\left(\cos \phi(t) \hat{I}_{p x}+\sin \phi(t) \hat{I}_{p y}\right)$,

where both the rf amplitude $\omega_{1}(t)$ and the rf phase $\phi(t)$ can be time-dependent due to the irradiation scheme. Under MAS, the radial part of the $\mathrm{rf}$ inhomogeneity will lead to additional modulations of $\omega_{1}(t)$ and $\phi(t)$ that are periodic with the rotor frequency. 
The spin-system Hamiltonian can be transformed into an interaction frame with respect to $\hat{\mathcal{H}}_{\mathrm{rf}}$ by the following:

$\hat{\tilde{\mathcal{H}}}(t)=\hat{U}_{\mathrm{rf}}^{-1}(t) \hat{\mathcal{H}}(t) \hat{U}_{\mathrm{rf}}(t)$.

The propagator characterizing the interaction frame transformation is given by the following:

$\hat{U}_{\mathrm{rf}}(t)=\hat{T} \exp \left(-i \int_{0}^{t} \hat{\mathcal{H}}_{\mathrm{rf}}\left(t^{\prime}\right) \mathrm{d} t^{\prime}\right)$,

where $\hat{T}$ is the Dyson time-ordering operator (Dyson, 1949) that ensures proper time-ordering of non-commuting operators in products. If the $\mathrm{rf}$ irradiation is periodic with $\tau_{\mathrm{m}}$, the $\hat{I}_{z}$ spin operators in Eq. (3) will transform according to the following:

$$
\begin{aligned}
\hat{\tilde{I}}_{z}(t) & =\sum_{\chi=x, y, z} a_{\chi}(t) \hat{I}_{\chi} \\
& =\sum_{\chi} \sum_{k} \sum_{\ell} a_{\chi}^{(k, \ell)} e^{i k \omega_{\mathrm{m}} t} e^{i \ell \omega_{\mathrm{eff}}} \hat{I}_{\chi},
\end{aligned}
$$

where the transformation behaviour of the Cartesian spin operator $\hat{I}_{z}$ is characterized by the Fourier coefficients $a_{\chi}^{(k, \ell)}$ (Scholz et al., 2009, 2010). These coefficients only depend on the rf irradiation scheme and are independent of the spin system details. Generally, the interaction frame trajectories of the spin operators are characterized by two basic frequencies, i.e. the modulation frequency of the pulse sequence $\omega_{\mathrm{m}}=\frac{2 \pi}{\tau_{\mathrm{m}}}$ and an additional effective nutation frequency that can be determined from the overall flip angle over one period of the pulse scheme $\omega_{\text {eff }}=\frac{\beta_{\text {eff }}}{\tau_{\mathrm{m}}}$. This additional effective field is zero if the propagator over a full cycle of the pulse sequence is unity (Tan et al., 2016).

The interaction frame Hamiltonian can thus be expanded as a Fourier series with three basic frequencies, as follows:

$\hat{\tilde{\mathcal{H}}}(t)=\sum_{n} \sum_{k} \sum_{\ell} \hat{\tilde{\mathcal{H}}}^{(n, k, \ell)} e^{i n \omega_{\mathrm{r}} t} e^{i k \omega_{\mathrm{m}} t} e^{i \ell \omega_{\mathrm{eff}} t}$,

with Fourier components $\hat{\tilde{\mathcal{H}}}^{(n, k, \ell)}$ as follows:

$$
\begin{aligned}
\hat{\tilde{\mathcal{H}}}^{(n, k, \ell)}= & \sum_{p=1}^{N} \omega_{p}^{(n)} \sum_{\chi} a_{\chi}^{(k, \ell)} \hat{I}_{p \chi}+\left[\sum_{p<q} \omega_{p q}^{(0)} \hat{\boldsymbol{I}}_{p} \cdot \hat{\boldsymbol{I}}_{q}\right] \\
& \times \delta_{n, 0} \cdot \delta_{k, 0} \cdot \delta_{\ell, 0}+\left[\sum_{p<q} 3 \cdot \omega_{p q}^{(n)} \sum_{\mu} \sum_{\chi} a_{\mu \chi}^{(k, \ell)}\right. \\
& \left.\times \hat{\boldsymbol{I}}_{p \mu} \hat{I}_{q \chi}-\left(\hat{\boldsymbol{I}}_{p} \cdot \hat{\boldsymbol{I}}_{q}\right) \cdot \delta_{k, 0} \cdot \delta_{\ell, 0}\right] \cdot\left(1-\delta_{n, 0}\right),
\end{aligned}
$$

where $\delta_{m, m^{\prime}}$ denotes the Kronecker delta. For convenience, the general two-spin Fourier coefficients $a_{\chi \mu}^{(k, \ell)}$ were defined. They can be computed as the convolution of single-spin coefficients as follows:

$a_{\mu \chi}^{(k, \ell)}=\sum_{k_{1}} \sum_{\ell_{1}} a_{\mu}^{\left(k_{1}, \ell_{1}\right)} a_{\chi}^{\left(k-k_{1}, \ell-\ell_{1}\right)}$
The scalar product of the $I$ spin vector operators remains time invariant under rf irradiation and can be incorporated into the $a_{\mu \mu}^{(0,0)}$ Fourier coefficients. In triple-mode Floquet theory, the first-order effective Hamiltonian is given by all contributions that satisfy the resonance condition, as follows:

$n_{0} \omega_{\mathrm{r}}+k_{0} \omega_{\mathrm{m}}+\ell_{0} \omega_{\mathrm{eff}}=0$,

and thus, the sum of non-resonant $\left(n_{0}=k_{0}=\ell_{0}=0\right)$ and resonant terms is as follows:

$\hat{\overline{\mathcal{H}}}_{\text {eff }}^{(1)}=\hat{\tilde{\mathcal{H}}}^{(0,0,0)}+\sum_{n_{0}, k_{0}, \ell_{0}} \hat{\tilde{\mathcal{H}}}^{\left(n_{0}, k_{0}, \ell_{0}\right)}$.

Analogously, in the following the second-order effective Hamiltonian is given by:

$\hat{\overline{\mathcal{H}}}_{\text {eff }}^{(2)}=\hat{\tilde{\mathcal{H}}}_{(2)}^{(0,0,0)}+\sum_{n_{0}, k_{0}, \ell_{0}} \hat{\tilde{\mathcal{H}}}_{(2)}^{\left(n_{0}, k_{0}, \ell_{0}\right)}$,

where:

$\hat{\tilde{\mathcal{H}}}_{(2)}^{\left(n_{0}, k_{0}, \ell_{0}\right)}=-\frac{1}{2} \sum_{\nu, \kappa, \lambda} \frac{\left[\hat{\tilde{\mathcal{H}}}^{\left(n_{0}-\nu, k_{0}-\kappa, \ell_{0}-\lambda\right)}, \hat{\tilde{\mathcal{H}}}^{(v, \kappa, \lambda)}\right]}{\nu \omega_{\mathrm{r}}+\kappa \omega_{\mathrm{m}}+\lambda \omega_{\mathrm{eff}}}$.

The summation is restricted to values of $v, \kappa$, and $\lambda$ for which $\nu \omega_{\mathrm{r}}+\kappa \omega_{\mathrm{m}}+\lambda \omega_{\text {eff }} \neq 0$ is satisfied in order to avoid singularities.

\subsubsection{Theoretical description including of inhomogeneity}

For spatial rf field distributions that do not have cylindrical rotation symmetry, MAS will lead to a periodic modulation of the rf field amplitude and phase experienced by a spin packet. At a given position, the general rf Hamiltonian, including these additional modulations, can be expressed as follows:

$$
\begin{aligned}
\hat{\mathcal{H}}_{\mathrm{rf}}(t)= & \omega_{1, \mathrm{rel}}(t) \cdot \omega_{1, \mathrm{nom}}(t)\left(\cos \left(\phi_{\mathrm{nom}}(t)+\phi_{\mathrm{rel}}(t)\right) \hat{I}_{x}\right. \\
& \left.+\sin \left(\phi_{\mathrm{nom}}(t)+\phi_{\mathrm{rel}}(t)\right) \hat{I}_{y}\right),
\end{aligned}
$$

where $\omega_{1 \text {, nom }}(t)$, and $\phi_{\text {nom }}(t)$ correspond to the nominal rf amplitude and phase, i.e. to the values corresponding to a perfectly homogeneous rf field. They are determined by the pulse scheme under investigation and are periodic with $\omega_{\mathrm{m}}=\frac{2 \pi}{\tau_{\mathrm{m}}}$. Deviations from these nominal values are introduced by the relative rf amplitude and phase $\omega_{1, \text { rel }}(t)$ and $\phi_{\text {rel }}(t)$ that are periodic with $\omega_{\mathrm{r}}=\frac{2 \pi}{\tau_{\mathrm{r}}}$. The overall period of the rf Hamiltonian will only be of finite length if the modulation frequency of the pulse scheme $\omega_{\mathrm{m}}$ and the rotor frequency $\omega_{\mathrm{r}}$ are commensurate:

$\omega_{\mathrm{m}}=\frac{\omega_{\mathrm{r}}}{c}$,

where $c$ corresponds to the number of rotor cycles required for the synchronization of the rf irradiation and the MAS 
rotation. Such a synchronization condition is generally fulfilled for rotor-synchronized pulse schemes such as most recoupling sequences (Nielsen et al., 2012). For irradiation schemes that are typically applied asynchronously to avoid resonance conditions, a careful selection of the synchronization condition is required. In principle, the treatment can be generalized to all cases where the largest common divisor of $\omega_{\mathrm{r}}$ and $\omega_{\mathrm{m}}$ is not too small. Such a synchronization is required to make the modulations of the rf field Hamiltonian by MAS cyclic over the basic repetition time of the sequence. The interaction frame Hamiltonian can then be written as follows:

$$
\begin{aligned}
\hat{\tilde{\mathcal{H}}}(t) & =\sum_{n=-2}^{2} \sum_{k} \sum_{\ell} \hat{\tilde{\mathcal{H}}}^{(n, k, \ell)} \underbrace{e^{i n \omega_{\mathrm{r}} t}}_{\text {MAS }} \underbrace{e^{i k \omega_{\mathrm{m}} t} e^{i \ell \omega_{\mathrm{eff}} t}}_{\text {rf irradiation }} \\
& =\sum_{n=-2}^{2} \sum_{k} \sum_{\ell} \hat{\tilde{\mathcal{H}}}^{(n, k, \ell)} e^{i(c n+k) \omega_{\mathrm{m}} t} e^{i \ell \omega_{\mathrm{eff}} t} \\
& =\sum_{n^{\prime}} \sum_{\ell} \hat{\tilde{\mathcal{H}}}^{\left(n^{\prime}, \ell\right)} e^{i n^{\prime} \omega_{\mathrm{m}} t} e^{i \ell \omega_{\mathrm{eff}} t},
\end{aligned}
$$

where the substitution $n^{\prime}=c \cdot n+k$ was used. The summation over the index $k$ runs from $-\infty$ to $+\infty$; the sum over $n^{\prime}$ in Eq. (23) is, thus, also unrestricted. The resulting interaction frame Hamiltonian is modulated with $\omega_{\mathrm{r}}=c \cdot \omega_{\mathrm{m}}$ due to the time dependence of the spatial part of the Hamiltonian during MAS (Fourier number $n$ ). The radial rf inhomogeneity leads to an additional modulation of the spin part with the rotor frequency. As the modulation frequency of the pulse sequence is commensurate with $\omega_{\mathrm{r}}$, these two modulations can be combined and a single Fourier number $n^{\prime}$ can be used. Therefore, the Hamiltonian is given by a Fourier series with only two basic frequencies $\left(\omega_{\mathrm{m}}\right.$ and $\left.\omega_{\text {eff }}\right)$, and the triplemode Floquet analysis is reduced to a bimodal treatment. Alternatively, one can continue with the triple-mode Floquet description and assume resonance conditions between $\omega_{\mathrm{m}}$ and $\omega_{\mathrm{r}}$. For resonant phenomena, the triple-mode Floquet approach is more suited since the additional effective field can lead to changes in the resonance conditions, leading to asynchronous sequences (Hellwagner et al., 2017; Tan et al., 2015). For non-resonant phenomena, both descriptions will give equivalent results. The periodic rf amplitude and phase modulations due to the radial inhomogeneity merely affect the interaction frame transformation, which is fully characterized by $\omega_{\mathrm{m}}$ and $\omega_{\mathrm{eff}}$, as long as these rf field modulations are periodic on the length of the interaction frame trajectory.

Possible resonance conditions for the bimodal interaction frame Hamiltonian of Eq. (23) are given by the following:

$n_{0}^{\prime} \omega_{\mathrm{m}}+\ell_{0} \omega_{\mathrm{eff}}=0$.

As effective fields due to the rf inhomogeneity will typically be small compared to $\omega_{\mathrm{r}}$, and $\ell$ is limited to a maximum value of two, it is reasonable to assume that only nonresonant terms will contribute to the effective Hamiltonian.
The second-order approximation of $\hat{\overline{\mathcal{H}}}_{\text {eff }}$ can, thus, be written as follows:

$\hat{\overline{\mathcal{H}}}_{\text {eff }}=\hat{\tilde{\mathcal{H}}}^{(0,0)}-\frac{1}{2} \sum_{\nu, \lambda} \frac{\left[\hat{\tilde{\mathcal{H}}}^{(-v,-\lambda)}, \hat{\tilde{\mathcal{H}}}^{(v, \lambda)}\right]}{\nu \omega_{\mathrm{m}}+\lambda \omega_{\text {eff }}}$

where the summation over $v$ and $\lambda$ is restricted to values satisfying $v \omega_{\mathrm{m}}+\lambda \omega_{\text {eff }} \neq 0$.

Each of the $\hat{\tilde{\mathcal{H}}}^{\left(n^{\prime}, \ell\right)}$ Fourier components in Eq. (23) is composed of a sum of several $\hat{\tilde{\mathcal{H}}}^{(n, k, \ell)}$ terms since there are multiple combinations of $n$ and $k$ resulting in the same $n^{\prime}$. As the index $n$ is limited to values between \pm 2 (limited by the rank of the spatial tensor), the $\hat{\tilde{\mathcal{H}}}^{\left(n^{\prime}, \ell\right)}$ are given by the following:

$\hat{\tilde{\mathcal{H}}}^{\left(n^{\prime}, \ell\right)}=\sum_{n=-2}^{2} \hat{\tilde{\mathcal{H}}}^{\left(n, n^{\prime}-c \cdot n, \ell\right)}$.

In the first-order approximation, the non-resonant contribution to the effective Hamiltonian is simply given by the $\hat{\tilde{\mathcal{H}}}^{(0,0)}$ Fourier component as follows:

$$
\begin{aligned}
\hat{\tilde{\mathcal{H}}}^{(0,0)}= & \hat{\tilde{\mathcal{H}}}^{(0,0,0)}+\sum_{\substack{n=-2 \\
n \neq 0}}^{2} \hat{\tilde{\mathcal{H}}}^{(n,-c \cdot n, 0)} \\
& =\sum_{p=1}^{N} \sum_{\chi} \sum_{n=-2}^{2} \omega_{p}^{(n)} a_{\chi}^{(-c \cdot n, 0)} \hat{I}_{p \chi} \\
& +\sum_{p<q} \sum_{\chi, \mu} \sum_{\substack{n=-2 \\
n \neq 0}}^{2} 3 \cdot \omega_{p q}^{(n)} a_{\chi \mu}^{(-c \cdot n, 0)} \hat{I}_{p \chi} \hat{I}_{q \mu} .
\end{aligned}
$$

In full analogy to Tan et al. (2016), the second-order effective Hamiltonian can be decomposed into three contributions from commutator cross-terms between chemical shift and dipolar-coupling terms,

$\hat{\tilde{\mathcal{H}}}_{(2)}^{(0,0)}=\hat{\overline{\mathcal{H}}}_{\mathrm{I} \otimes \mathrm{I}}+\hat{\overline{\mathcal{H}}}_{\mathrm{I} \otimes \mathrm{II}}+\hat{\overline{\mathcal{H}}}_{\mathrm{II} \otimes \mathrm{II}}$,

where:

$\hat{\overline{\mathcal{H}}}_{\mathrm{I} \otimes \mathrm{I}}=\sum_{p=1}^{N} \sum_{n_{1}=-2}^{2} \sum_{n_{2}=-2}^{2} \sum_{\chi} \frac{-i}{2} \omega_{p}^{\left(n_{1}\right)} \omega_{p}^{\left(n_{2}\right)} q_{\chi}^{\left(n_{1}, n_{2}\right)} \hat{I}_{p \chi}$,
$\hat{\mathcal{H}}_{\mathrm{I} \otimes \mathrm{II}}=\sum_{p \neq q} \sum_{\chi, \mu} \sum_{n_{1}=-2}^{2} \sum_{\substack{n_{2}=-2 \\ n_{2} \neq 0}}^{2} \frac{-3 i}{2} \omega_{p}^{\left(n_{1}\right)} \omega_{p q}^{\left(n_{2}\right)} q_{\chi, \mu}^{\left(n_{1}, n_{2}\right)} \hat{I}_{p \chi} \hat{I}_{q \mu}$,

$$
\begin{aligned}
\hat{\overline{\mathcal{H}}}_{\mathrm{II} \otimes \mathrm{II}}= & \sum_{p \neq q} \sum_{\chi} \sum_{n_{1}, n_{2}}-\frac{9 i}{8} \omega_{p q}^{\left(n_{1}\right)} \omega_{p q}^{\left(n_{2}\right)} p_{\chi}^{\left(n_{1}, n_{2}\right)} \hat{I}_{p \chi} \\
& +\sum_{p \neq q \neq o \chi, \mu, \xi} \sum_{n_{1}, n_{2}}-\frac{9 i}{2} \omega_{p q}^{\left(n_{1}\right)} \omega_{q o}^{\left(n_{2}\right)} p_{\mu \chi \xi}^{\left(n_{1}, n_{2}\right)} \\
& \times \hat{I}_{p \mu} \hat{I}_{q \chi} \hat{I}_{o \xi} .
\end{aligned}
$$


The second-order scaling factors $q_{\chi}^{\left(n_{1}, n_{2}\right)}, q_{\chi \mu}^{\left(n_{1}, n_{2}\right)}, p_{\chi}^{\left(n_{1}, n_{2}\right)}$ and $p_{\mu \chi \xi}^{\left(n_{1}, n_{2}\right)}$ for $\chi=x$ are given by the following:

$$
\begin{aligned}
q_{x}^{\left(n_{1}, n_{2}\right)}= & \sum_{\nu, \lambda} \frac{1}{v \omega_{\mathrm{m}}+\lambda \omega_{\mathrm{eff}}}\left(a_{y}^{\left(-\nu-c \cdot n_{1},-\lambda\right)} a_{z}^{\left(\nu-c \cdot n_{2}, \lambda\right)}\right. \\
& \left.-a_{z}^{\left(-\nu-c \cdot n_{1},-\lambda\right)} a_{y}^{\left(\nu-c \cdot n_{2}, \lambda\right)}\right) \\
q_{x, \mu}^{\left(n_{1}, n_{2}\right)}= & \sum_{\nu, \lambda} \frac{1}{\nu \omega_{\mathrm{m}}+\lambda \omega_{\mathrm{eff}}}\left(a_{y}^{\left(-\nu-c \cdot n_{1},-\lambda\right)} a_{z \mu}^{\left(\nu-c \cdot n_{2}, \lambda\right)}\right. \\
& -a_{z}^{\left(-v-c \cdot n_{1},-\lambda\right)} a_{y \mu}^{\left(\nu-c \cdot n_{2}, \lambda\right)} \\
& -a_{y}^{\left(\nu-c \cdot n_{1}, \lambda\right)} a_{z \mu}^{\left(-v-c \cdot n_{2},-\lambda\right)} \\
& \left.+a_{z}^{\left(v-c \cdot n_{1}, \lambda\right)} a_{y \mu}^{\left(-v-c \cdot n_{2},-\lambda\right)}\right)
\end{aligned}
$$

$$
\begin{aligned}
p_{x}^{\left(n_{1}, n_{2}\right)}= & \sum_{\mu} \sum_{\nu, \lambda} \frac{1}{v \omega_{\mathrm{m}}+\lambda \omega_{\mathrm{eff}}}\left(a_{y \mu}^{\left(-\nu-c \cdot n_{1},-\lambda\right)} a_{z \mu}^{\left(\nu-c \cdot n_{2}, \lambda\right)}\right. \\
& \left.-a_{z \mu}^{\left(-v-c \cdot n_{1},-\lambda\right)} a_{y \mu}^{\left(\nu-c \cdot n_{2}, \lambda\right)}\right)
\end{aligned}
$$

$$
\begin{aligned}
p_{\mu x \xi}^{\left(n_{1}, n_{2}\right)}= & \sum_{\nu, \lambda} \frac{1}{v \omega_{\mathrm{m}}+\lambda \omega_{\mathrm{eff}}}\left(a_{\mu y}^{\left(-v-c \cdot n_{1},-\lambda\right)} a_{z \xi}^{\left(\nu-c \cdot n_{2}, \lambda\right)}\right. \\
& -a_{\mu y}^{\left(\nu-c \cdot n_{1}, \lambda\right)} a_{z \xi}^{\left(-v-c \cdot n_{2},-\lambda\right)} \\
& -a_{\mu z}^{\left(-v-c \cdot n_{1},-\lambda\right)} a_{y \xi}^{\left(\nu-c \cdot n_{2}, \lambda\right)} \\
& \left.+a_{\mu z}^{\left(v-c \cdot n_{1}, \lambda\right)} a_{y \xi}^{\left(-v-c \cdot n_{2},-\lambda\right)}\right)
\end{aligned}
$$

Similar expressions result for $\chi=y$ and $z$ and can be found in the Supplement (Sect. S1).

All first- and second-order scaling factors can be obtained from the Fourier coefficients $a_{\chi}^{(k, \ell)}$ characterizing the rf interaction frame trajectory of the Cartesian spin operator $\hat{I}_{z}$ and, thus, do not depend on the details of the spin system. The effects of the additional rf field modulations due to the radial contribution to the rf inhomogeneity will lead to changes in the interaction frame trajectories and, therefore, changes in the scaling factors for the effective Hamiltonians compared to those calculated assuming a perfectly homogeneous rf field.

\section{Methods and materials}

\subsection{Numerical simulations}

The effect of the rf field inhomogeneity on common solidstate NMR pulse sequences was investigated by numerical simulations in the usual rotating frame using the GAMMA spin-simulation environment (Smith et al., 1994). Unless otherwise noted, spin dynamics were simulated at a $B_{0}$ field of $14.1 \mathrm{~T}$, corresponding to a proton resonance frequency of $600 \mathrm{MHz}$. Powder averaging was implemented according to the Zaremba-Conroy-Wolfsberg (ZCW; Cheng et al., 1973) scheme, using between 100 and 10000 crystallite orientations. A summary of the simulation parameters, such as MAS frequencies and nominal rf field strengths for the individual pulse sequences that were investigated, is given in the Supplement (Table S1).

For all experimental schemes treated here, the general form of the time-dependent rf field Hamiltonian is given by Eq. (19). Deviations from the nominal rf amplitude and phase due to the $\mathrm{rf}$ inhomogeneity are introduced by the relative amplitude and phase, denoted by $\omega_{1, \text { rel }}(\boldsymbol{r}, t)$, and $\phi_{\text {rel }}(\boldsymbol{r}, t)$, respectively. These parameters depend on the position of the crystallite in the sample space $\boldsymbol{r}$ and the rotor orientation. Under MAS, they are modulated by the rotor frequency. The time-dependent trajectories were computed numerically (see Sect. 2) and are given as an input to the numerical simulations.

Simulations were performed for volume elements of the $r z$ plane indicated in yellow in Fig. 1a, with an initial orientation of $\vartheta_{0}=0^{\circ}$. For nutation experiments (see Sect. 5.1), several $\vartheta_{0}$ values were considered, as the spin dynamics are, in principle, dependent on $\vartheta_{0}$ due to the non-commuting Hamiltonians at different time points during a rotor period. The sample space was restricted to the length of the coil along the rotor axis (indicated by dashed lines in Fig. 1b). Potential effects of the radial inhomogeneity should be similar for samples exceeding the length of the coil but might be more pronounced since the magnitude of rf amplitude and phase modulations increases towards the rotor edges (see Fig. 2). Simulation results of the individual volume elements were summed up during data processing and weighted with $r$ to account for the increase in volume with radial distance. The coil sensitivity (reciprocity theorem; Hoult and Richards, 1976; Tošner et al., 2017) was taken into account by additional weighting of each $r z$ element with the average relative rf amplitude over a rotor cycle $\bar{\omega}_{1, \text { rel }}(\boldsymbol{r})$.

In order to separate the effect of the static rf field inhomogeneity from time-dependent effects due to amplitude and phase modulation arising from sample rotations, the spin dynamics were simulated under different conditions. Amplitude and phase modulations were considered separately and either treated as time dependent or as the static average over a rotor period. The four following cases considered in this work are denoted as $\mathrm{C} 1-\mathrm{C} 4$, where:

- C1 - time-averaged constant amplitude and zero phase; $\bar{\omega}_{1, \text { rel }}(\boldsymbol{r})=\frac{1}{\tau_{\mathrm{r}}} \int_{t=0}^{\tau_{\mathrm{r}}} \omega_{1, \mathrm{rel}}(\boldsymbol{r}, t) \mathrm{d} t, \phi_{\text {rel }}(\boldsymbol{r}, t)=0$

- C2 - time-dependent amplitude and zero phase; $\omega_{1, \mathrm{rel}}(\boldsymbol{r}, t), \phi_{\text {rel }}(\boldsymbol{r}, t)=0$

- C3 - time-averaged constant amplitude and timedependent phase;

$\bar{\omega}_{1, \mathrm{rel}}(\boldsymbol{r})=\frac{1}{\tau_{\mathrm{r}}} \int_{t=0}^{\tau_{\mathrm{r}}} \omega_{1, \mathrm{rel}}(\boldsymbol{r}, t) \mathrm{d} t, \phi_{\mathrm{rel}}(\boldsymbol{r}, t)$ 
Table 1. Summary of the treatment of the relative rf amplitude and phase for the four cases $\mathrm{C} 1-\mathrm{C} 4$.

\begin{tabular}{lllll}
\hline & $\mathrm{C} 1$ & $\mathrm{C} 2$ & $\mathrm{C} 3$ & $\mathrm{C} 4$ \\
\hline Amplitude & $\bar{\omega}_{1, \mathrm{rel}}(\boldsymbol{r})$ & $\omega_{1, \mathrm{rel}}(\boldsymbol{r}, t)$ & $\bar{\omega}_{1, \mathrm{rel}}(\boldsymbol{r})$ & $\omega_{1, \mathrm{rel}}(\boldsymbol{r}, t)$ \\
Phase & 0 & 0 & $\phi_{\mathrm{rel}}(\boldsymbol{r}, t)$ & $\phi_{\mathrm{rel}}(\boldsymbol{r}, t)$ \\
\hline
\end{tabular}

- C4 - time-dependent amplitude and time-dependent phase;

$\omega_{1, \mathrm{rel}}(\boldsymbol{r}, t), \phi_{\mathrm{rel}}(\boldsymbol{r}, t)$.

Simulations with the time-averaged constant phase were not performed as constant phase offsets are small (maximum of less than $5^{\circ}$ ), and the absolute phase of the rf irradiation has no influence on the outcome of an experiment. For reference, Table 1 summarizes the treatment of amplitude and phase modulations for these four cases.

\subsection{Experiment}

Experiments were performed on a $500 \mathrm{MHz}$ Bruker Avance III HD NMR spectrometer equipped with a Bruker $1.9 \mathrm{~mm}$ triple-resonance MAS probe in double-resonance configuration at a temperature of $285 \mathrm{~K}$. All powdered samples (natural-abundance glycine and natural-abundance L-histidine $\cdot \mathrm{HCl} \cdot \mathrm{H}_{2} \mathrm{O}$ ) were purchased from commercial sources and used without further purification. Nutation spectra of glycine were recorded as two-dimensional experiments without sign discrimination in $t_{1}$ (simple sine amplitude modulation) at MAS frequencies of 15 and $30 \mathrm{kHz}$. The nominal rf field amplitude was calibrated to $100 \mathrm{kHz}$ using a nutation spectrum. Spectra were recorded with $512 t_{1}$ increments with 12 scans each and a time increment for the nutation pulse of $2.5 \mu$ s. The spectral width in the direct dimension was set to $100 \mathrm{kHz}$, and 1024 complex data points were acquired. MATLAB (The MathWorks Inc., Natick, MA, USA) was used for data processing using a cosine-squared window function. The two-dimensional proton-proton correlation spectra of L-histidine with frequency-switched LeeGoldburg (FSLG) decoupling (Bielecki et al., 1989, 1990; Mote et al., 2016) in the indirect dimension were recorded at MAS frequencies of 14 and $28 \mathrm{kHz}$. Spectra were acquired with $512 t_{1}$ increments with eight scans each and time increments between 43.2 and $48 \mu$ s. States-type (States et al., 1982) data acquisition was used for phase-sensitive detection and sign discrimination in $t_{1}$. The spectral width in the direct dimension was set to $200 \mathrm{kHz}$, and 1024 complex data points were recorded. Nutation-frequency-selective I-BURP2 (Geen and Freeman, 1991) pulses in the spin lock frame were used for sample restriction (Aebischer et al., 2020). The rf field amplitudes were calibrated using a nutation spectrum and set to $100 \mathrm{kHz}$ during hard pulses and spin lock. The FSLG decoupling was implemented using shaped pulses with a time resolution of $100 \mathrm{~ns}$ for the phase ramp. Shape files with 80, 108, and 160 points were used, corresponding to nutation frequencies about the effective field of 250 , 185.2 , and $125 \mathrm{kHz}$. The carrier was placed outside the spectral region of interest, and its position is indicated by an arrow. Spectra were processed in MATLAB with zero-filling to $4096 \times 4096$ data points and the application of a cosinesquared apodization. The 1D spectra shown were obtained by summation over the relevant spectral region in $\omega_{2}$. Frequency axes in parts per million (ppm) were determined by comparison of the peak positions observed for the $\alpha$ and $\delta^{2}$ proton resonances in histidine with those found in the literature (Mithu et al., 2013).

\section{Results and discussion}

In this section, we discuss how a number of common solidstate NMR experiments are affected by the MAS timemodulated radio-frequency fields. This is done by analytical calculations based on the Floquet description presented in Sect. 3.1, numerical simulations, and, in some cases, using experimental data.

\subsection{Nutation spectroscopy}

\subsubsection{Numerical simulations and experimental results}

Nutation spectra represent a simple method for characterizing the rf field distribution in the sample. Such spectra were simulated for one-spin systems, and the rf inhomogeneity was included in the rf Hamiltonian of Eq. (19), setting the nominal phase of the rf field to zero corresponding to if irradiation along the $x$ axis. The nominal $\mathrm{rf}$ field amplitude $v_{1, \text { nom }}$ was set to $100 \mathrm{kHz}$, and the four cases $\mathrm{C} 1-\mathrm{C} 4$ (see Table 1) were studied. As only isotropic spin interactions were considered, simulations were performed for a single crystallite orientation.

Simulated nutation spectra, using the rf field profiles of the 3.2 and $1.3 \mathrm{~mm}$ MAS probes at different spinning frequencies (15 and $30 \mathrm{kHz}$, respectively), are shown in Fig. 3. The overall nutation profile of the $1.3 \mathrm{~mm}$ probe is narrower, indicating a more homogeneous rf field distribution inside the coil and, thus, a less pronounced drop-off of the static rf field amplitude along the rotor axis. Phase modulation of the $\mathrm{rf}$ field (C3 and C4) leads to sidebands at $0 \pm m \cdot v_{\mathrm{r}}(m=1,2$ are visible). The intensity of these sidebands increases with increasing MAS frequency. Amplitude modulation of the applied rf field, on the other hand (C2 and $\mathrm{C} 4)$, leads to sidebands at $v_{1} \pm m \cdot v_{\mathrm{r}}$ (only $m=1$ visible). These sidebands are significantly weaker than those arising due to phase modulations, and their intensity increases with decreasing spinning frequency. The reduced intensity of these amplitude modulation sidebands can be explained by the fact that their position depends on the magnitude of the static rf field amplitude and, thus, varies depending on the position within the sample space. Both types of sidebands are weaker in the $1.3 \mathrm{~mm}$ rf 
(a)

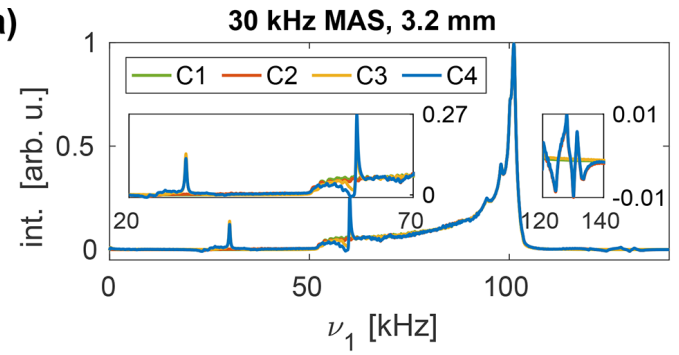

(b)

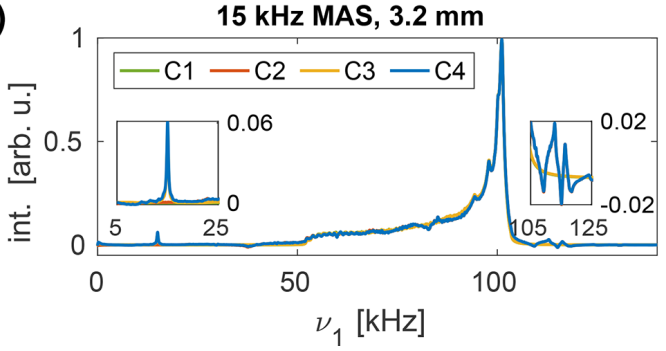

(c)

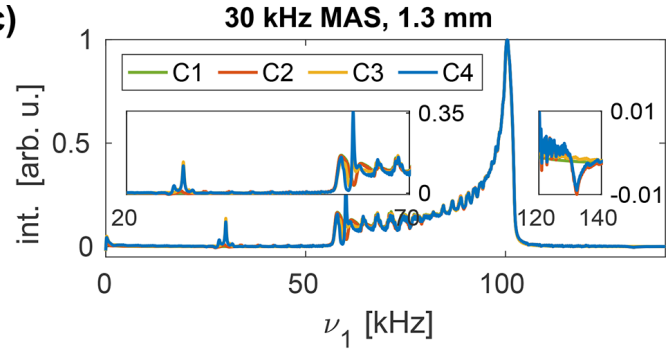

(d)

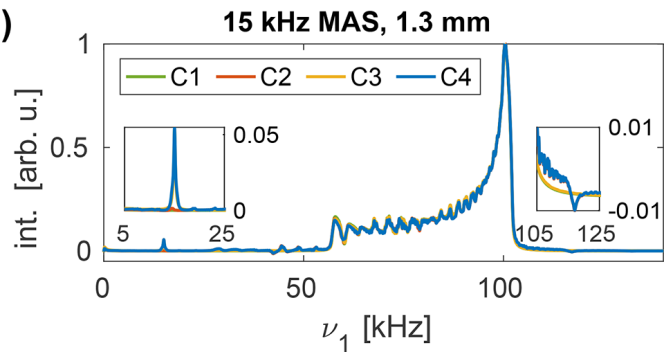

Figure 3. Simulated nutation spectra at a resonance frequency of $600 \mathrm{MHz}$ for the $3.2 \mathrm{~mm}$ MAS probe (a, b) and the $1.3 \mathrm{~mm}$ MAS probe (c, d) for a nominal rf amplitude of $100 \mathrm{kHz}$. A spinning frequency of $30 \mathrm{kHz}(\mathbf{a}, \mathbf{c})$ and $15 \mathrm{kHz}(\mathbf{b}, \mathbf{d})$ was assumed for the spectra. Sidebands at $0 \pm m \cdot v_{\mathrm{r}}$ arise when rf phase modulations are taken into account ( $\mathrm{C} 3$ and $\mathrm{C} 4$ ), while those at $v_{1} \pm m \cdot v_{\mathrm{r}}$ occur if amplitude modulations are present ( $\mathrm{C} 2$ and $\mathrm{C} 4)$. The amplitude modulation sidebands increase in intensity at lower MAS frequencies, whereas the phase modulation sidebands are attenuated. Both sideband families are less intense in the $1.3 \mathrm{~mm}$ probe, and the overall nutation profile is narrower, indicating an improved homogeneity of the rf distribution inside the coil.

profile, indicating that $\mathrm{rf}$ amplitude and phase modulations are less pronounced in comparison to the $3.2 \mathrm{~mm}$ rf profile. The phases of the sidebands depend on the initial position $\vartheta_{0}$ of the simulated $r z$ plane (see Fig. S1 in the Supplement). However, the obtained spectra are very similar for all initial orientations, and no significant influence of $\vartheta_{0}$ on the effects of the radial rf inhomogeneity has been observed.

Experimental ${ }^{1} \mathrm{H}$ nutation spectra of natural-abundance glycine measured at a proton resonance frequency of $500 \mathrm{MHz}$, using a Bruker $1.9 \mathrm{~mm}$ MAS probe, are shown in Fig. 4 for MAS frequencies of $30 \mathrm{kHz}$ (Fig. $4 \mathrm{a}$ ) and $15 \mathrm{kHz}$ (Fig. 4b). As was observed in the simulated nutation spectra (see Fig. 3), sidebands at $0 \pm m \cdot v_{\mathrm{r}}$ due to rf phase modulations are visible in the experimental spectra. Moreover, sidebands at $v_{1, \mathrm{nom}} \pm m \cdot v_{\mathrm{r}}$ are visible that replicate the shape of the main nutation profile at $100 \mathrm{kHz}$. At a lower MAS frequency, these sidebands increase in intensity, whereas those at multiples of the rotor frequency are attenuated. In the simulated spectra shown in Fig. 3, the sidebands at 115 and $130 \mathrm{kHz}$ were significantly weaker and did not have the same shape as the overall nutation profile. As is shown in Fig. S2, no such sidebands are observed in the experimental nutation spectra of natural-abundance adamantane (see Fig. S2a), indicating that they arise from the MAS modulation of anisotropic interactions. This is confirmed by the simulated nutation spectra obtained for a dipolar-coupled two-spin system (see Fig. S2b), where strong sidebands at $v_{1} \pm v_{r}$ are obtained that nicely replicate the shape of the main nutation profile for all four cases $(\mathrm{C} 1-\mathrm{C} 4)$. (a)

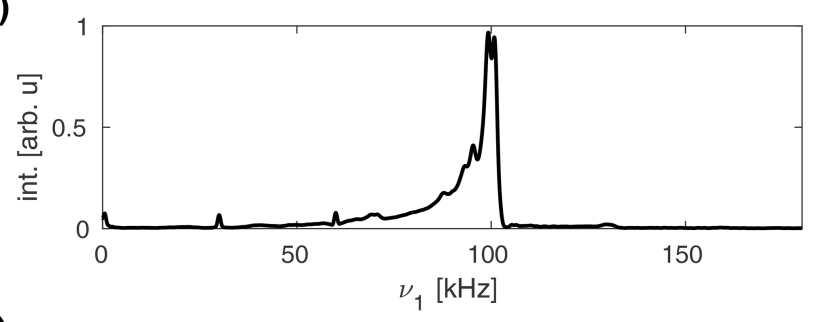

(b)

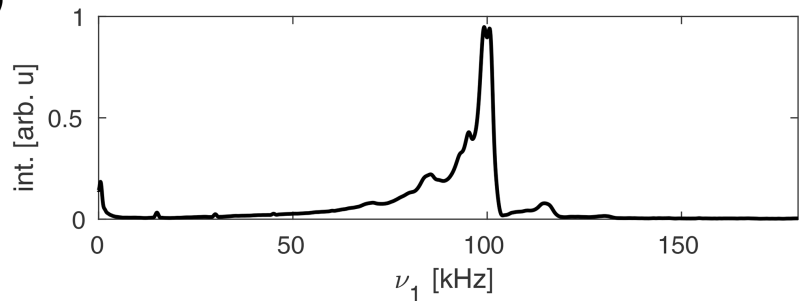

Figure 4. Experimental ${ }^{1} \mathrm{H}$ nutation spectra of natural-abundance glycine recorded at a proton resonance frequency of $500 \mathrm{MHz}$ in a Bruker $1.9 \mathrm{~mm}$ MAS probe spinning at $30 \mathrm{kHz}$ (a) and $15 \mathrm{kHz}$ (b). The nominal rf amplitude was set to $100 \mathrm{kHz}$, as determined using a nutation spectrum. Sidebands due to rf phase modulations at $0 \pm m \cdot v_{\mathrm{r}}$ for $m=1,2$ are visible in both spectra but have lower intensity at slower MAS, as expected from the simulations (see Fig. 3). Much broader sidebands that replicate the shape of the overall nutation profile are visible at $v_{1, \text { nom }} \pm m \cdot v_{\mathrm{r}}$ for $m=1$. Their intensity increases significantly at the lower MAS frequency. 


\subsubsection{Floquet analysis}

Phase modulation of the rf field leads to non-commuting terms in the rf Hamiltonian at different points in time, thus prohibiting an analytical determination of the time evolution of the magnetization during the nutation experiment. However, insight can be gained from the interaction frame trajectory of spin operators that can be computed numerically. For the $\hat{I}_{z}$ spin operator, this trajectory can be expanded as follows:

$\hat{\tilde{I}}_{z}(t)=a_{x}(t) \hat{I}_{x}+a_{y}(t) \hat{I}_{y}+a_{z}(t) \hat{I}_{z}$.

Fourier analysis of the time-dependent $a_{\chi}(t)$ coefficients then yields the frequency components present in the nutation spectrum. Such interaction frame trajectories of the $\hat{I}_{z}$ spin operator with rf irradiation along the $x$ axis were computed numerically in MATLAB with a time resolution of $50 \mathrm{~ns}$. A nominal rf field strength of $100 \mathrm{kHz}$ was chosen and a MAS frequency of $30 \mathrm{kHz}$ assumed. The effects of MAS timedependent $\mathrm{rf}$ amplitude and phase modulations were studied separately. Modulations were modelled as Fourier series (see also Eqs. 1 and 2), and magnitude $A_{n}^{(\mathrm{A} / \mathrm{P})}$ and phase $\phi_{n}^{(\mathrm{A} / \mathrm{P})}$ coefficients were given as input.

Absolute values and phases of the $a_{\chi}(t)$ coefficients are shown in Figs. 5a and $\mathrm{b}$ as a function of the magnitude of amplitude modulations with $\omega_{\mathrm{r}}$ and $2 \cdot \omega_{\mathrm{r}}\left(A_{1}^{(\mathrm{A})}\right.$ and $\left.A_{2}^{(\mathrm{A})}\right)$. The static amplitude offset $A_{0}^{(\mathrm{A})}$ was set to 1 , and all $A_{n}^{(\mathrm{A})}$ with $n \geq 3$ were set to zero. No phase modulation was taken into account. Amplitude modulation with the rotor frequency (Fig. 5a) leads to sidebands at $v_{1} \pm m \cdot v_{\mathrm{r}}$, with $m$ being any integer, whereas amplitude modulation with $2 \cdot \omega_{\mathrm{r}}$ (Fig. 5b) leads to sidebands at $v_{1} \pm 2 m \cdot v_{\mathrm{r}}$. The intensity of these sidebands increases with the magnitude of the modulation in both cases. However, sidebands arising from rf amplitude modulation with the base frequency $\omega_{\mathrm{r}}$ are significantly stronger. The phase of the amplitude modulation $\phi_{n}^{(\mathrm{A})}$ for the spectra in Fig. 5 was set to zero as it only influences the phases of the centre band and sidebands. Static amplitude offsets $A_{0}^{(\mathrm{A})} \neq 1$ will simply shift the entire spectrum. Figure $5 \mathrm{c}$ and $\mathrm{d}$ show the absolute values and phases of $a_{\chi}(t)$ for phase modulations with $\omega_{\mathrm{r}}$ and $2 \cdot \omega_{\mathrm{r}}\left(A_{1}^{(\mathrm{P})}\right.$ and $\left.A_{2}^{(\mathrm{P})}\right)$. The static phase offset $A_{0}^{(\mathrm{P})}$ and all $A_{n}^{(\mathrm{A})}$ with $n \geq 3$ were set to zero. No rf amplitude modulations were taken into account. The frequency range shown in the figure was limited to $0-90 \mathrm{kHz}$ since $\mathrm{rf}$ phase modulations lead to sidebands at $0 \pm n \cdot v_{\mathrm{r}}$ that are significantly less intense than the main band at the nominal rf amplitude $\left(v_{1}=100 \mathrm{kHz}\right)$. In contrast to the sidebands observed for $\mathrm{rf}$ amplitude modulation, phase modulation with $n \cdot \omega_{\mathrm{r}}$ exclusively leads to sidebands at $0 \pm n \cdot v_{\mathrm{r}}$. Compared to the sidebands arising from amplitude modulation, the intensities of these phase modulation sidebands are considerably lower. Their intensity increases with the Fourier number $n$ of the modulation (sidebands arising from $A_{2}^{(\mathrm{P})}$ are more intense than the ones from $\left.A_{1}^{(\mathrm{P})}\right)$. The phase of the modulation $\left(\phi_{n}^{(\mathrm{P})}\right)$ was set to zero again, as it only affects the phase of the $a_{\chi}(t)$ coefficients (see also Fig. S1).

These results are in good agreement with the simulated and experimental nutation spectra shown in Figs. 3 and 4, where two separate families of sidebands arose for $\mathrm{rf}$ amplitude and phase modulation. As described earlier, the higher intensity observed for phase modulation sidebands in these spectra can be explained by the overall larger magnitude of the phase modulations and the independence of the sideband position from the average rf field amplitude. The position of the amplitude modulation sidebands, on the other hand, shifts with the average static rf field amplitude, leading to a broadening of the sidebands.

We have also looked at the effects of the radial rf field inhomogeneity in the context of spin lock experiments which is closely related to the nutation experiment. This connects to the first experimental observation of such effects in rotary resonance recoupling experiments, where additional peaks in the centre of the expected dipolar doublet have been observed. This was attributed to time-dependent phase modulations (Levitt et al., 1988). A more detailed study of this experiment is described in Sect. S4.

\subsection{Cross-polarization}

Hartmann-Hahn cross-polarization (Hartmann and Hahn, 1962; Stejskal et al., 1977) is probably the most ubiquitous pulse sequence element in solid-state NMR. Under MAS, the sum, or difference, of the two rf field amplitudes has to be matched to an integer multiple of the spinning frequency as follows:

$\omega_{1 \mathrm{~S}} \pm \omega_{1 \mathrm{I}}=n \cdot \omega_{\mathrm{r}} \quad n= \pm 1, \pm 2$.

Due to the rf field inhomogeneity across the sample, this condition cannot be fulfilled simultaneously in the entire sample volume, and only certain parts of the sample will participate in the polarization transfer, thus decreasing the resulting signal intensity. One popular strategy to overcome this volume selectivity is ramped-amplitude cross-polarization (Metz et al., 1994) or the adiabatic modulation of the rf field amplitude during the contact time (Hediger et al., 1995, see Sect. S5 for more details). In this work $\hat{I}_{x} \rightarrow \hat{S}_{x}$ magnetization transfers at the $n=1$ zero-quantum matching conditions in heteronuclear CN, HN, and HC two-spin systems, were simulated for standard, ramped-amplitude, and adiabatic-passage $\mathrm{CP}$ experiments for the $3.2 \mathrm{~mm}$ MAS probe at a proton resonance frequency of $600 \mathrm{MHz}$. A MAS frequency of $20 \mathrm{kHz}$ was assumed, and the nominal $\mathrm{rf}$ fields and contact times were set for $\mathrm{CN}$ as follows: $85 \mathrm{kHz}$ on $\mathrm{C}, 65 \mathrm{kHz}$ on $\mathrm{N}$, and $5 \mathrm{~ms}$. The nominal rf fields and contact times were set for $\mathrm{HN}$ as follows: $70 \mathrm{kHz}$ on $\mathrm{H}, 50 \mathrm{kHz}$ on $\mathrm{N}$, and $1 \mathrm{~ms}$. The nominal $\mathrm{rf}$ fields and contact times were set for $\mathrm{HC}$ as follows: $90 \mathrm{kHz}$ on $\mathrm{H}, 70 \mathrm{kHz}$ on $\mathrm{C}$, and $1 \mathrm{~ms}$. The anisotropy of the dipolarcoupling tensor $\delta_{\mathrm{IS}}=-2 \frac{\mu_{0} \hbar_{\gamma_{I} \gamma_{S}}}{4 \pi r_{\mathrm{IS}}^{3}}$ was estimated from aver- 

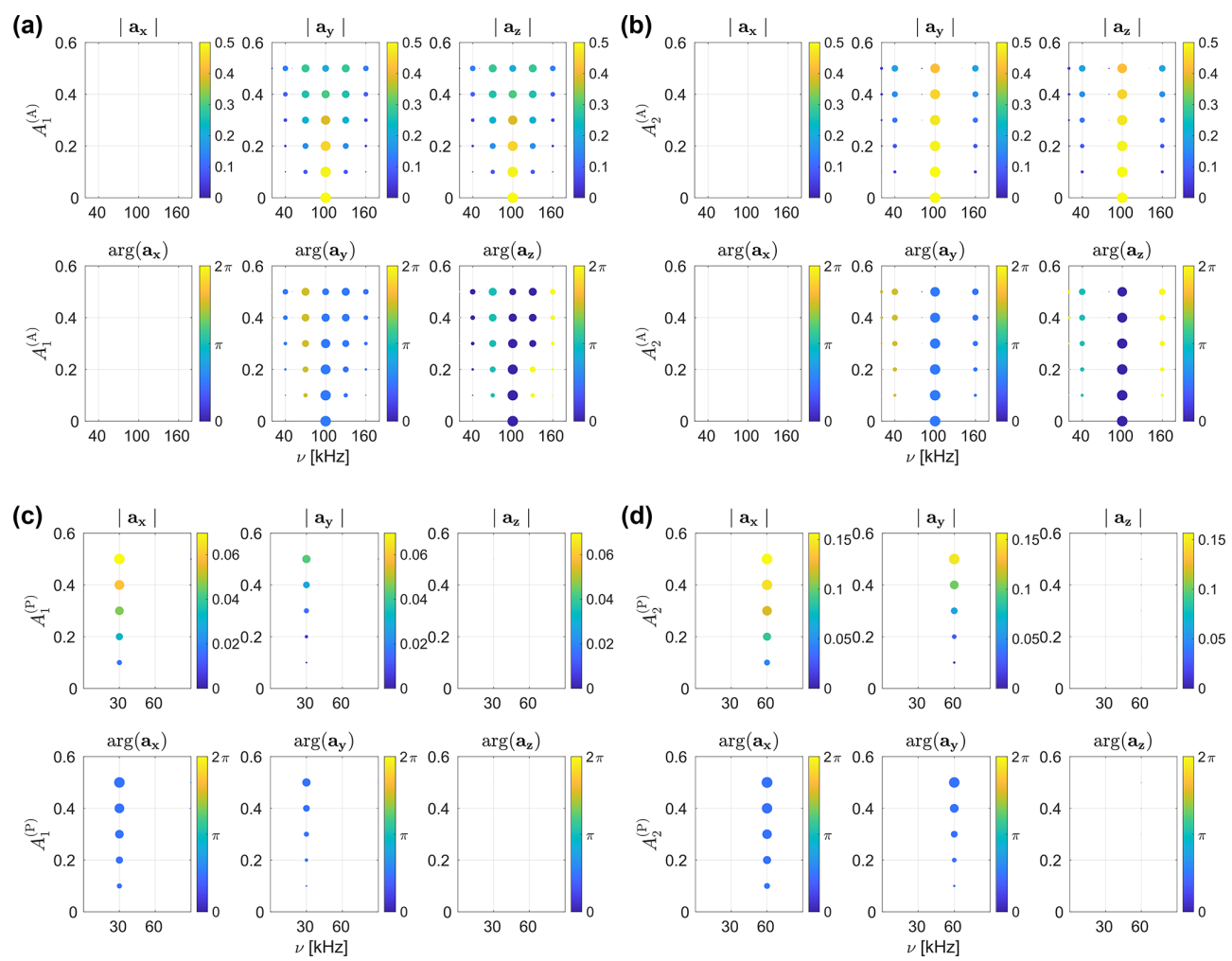

Figure 5. Absolute values and phases of the $a_{\chi}(t)$ coefficients characterizing the interaction frame trajectory of the $\hat{I}_{z}$ operator during a nutation experiment with rf irradiation along the $x$ axis. A nominal rf field strength of $100 \mathrm{kHz}$ and a MAS frequency of $30 \mathrm{kHz}$ was assumed. The magnitude and phase of the coefficients are shown as a function of the $A_{n}^{(\mathrm{A})}$ (a, b) and $A_{n}^{(\mathrm{P})}$ (c, d) magnitude coefficients for rf amplitude modulations, with $\omega_{\mathrm{r}}(\mathbf{a})$ and $2 \cdot \omega_{\mathrm{r}}(\mathbf{b})$, as well as phase modulations with $\omega_{\mathrm{r}}(\mathbf{c})$ and $2 \cdot \omega_{\mathrm{r}}(\mathbf{d})$. Sidebands due to the MAS modulation of the rf amplitude at $v_{1} \pm m \cdot v_{\mathrm{r}}$, with $m$ being any integer for modulations with $\omega_{\mathrm{r}}\left(A_{1}^{(\mathrm{A})}\right)$, and any even integer for modulations with $2 \cdot \omega_{\mathrm{r}}\left(A_{2}^{(\mathrm{A})}\right)$ is observed. The intensity of these sidebands increases with the strength of the modulation. For rf phase modulations, sidebands at $0 \pm n \cdot v_{\mathrm{r}}$ arise for modulations with $n \cdot \omega_{\mathrm{r}}$. The intensity of these sidebands increases with the strength of the modulation and considerably higher intensities are observed for higher $n$ (note the different scaling of the colour bars for $A_{1}^{(\mathrm{P})}$ and $A_{2}^{(\mathrm{P})}$ in $\mathbf{c}$ and $\mathbf{d}$ ). The frequency axes in $(\mathbf{c}, \mathbf{d})$ were restricted to $0-90 \mathrm{kHz}$ since the phase modulation sidebands are significantly weaker than the main band contribution to $a_{z}(t)$ at the nominal rf amplitude of $100 \mathrm{kHz}$.

age bond lengths, and $\frac{\delta_{\mathrm{IS}}}{2 \pi}$ values of $1.9,25$, and $-46 \mathrm{kHz}$ were used for the $\mathrm{CN}, \mathrm{HN}$, and $\mathrm{HC}$ simulations, respectively. Chemical shifts as well as $J$ coupling constants were set to zero. The simulations were performed with a time resolution of $250 \mathrm{~ns}$, and the $x$ magnetization of both the source and the destination spin was detected every $5 \mu$ s. Powder averaging was performed over 1154 crystallite orientations.

The simulated time evolution of the spin-locked $x$ magnetization on both spins for the cases $\mathrm{C} 1-\mathrm{C} 4$ (see Table 1) is shown in Fig. 6 for all three spin pairs. The rf amplitude on the source spin was either kept constant (left-hand column), modulated with a linear ramp (middle column), or used a tangential modulation (right-hand column; see Fig. S4 for more details). In $\mathrm{CN}$ spin pairs, a tangential modulation of the rf amplitude on one of the spins leads to a significant improvement of the transfer efficiency (up to $35 \%$ ) compared to both the standard and the ramped amplitude CP experiment (less than $20 \%$ ). For spin pairs with stronger dipolar couplings, such as $\mathrm{HN}$ and $\mathrm{HC}$, both the ramped amplitude $\mathrm{CP}$ and the adiabatic passage $\mathrm{CP}$ lead to similar transfer efficiencies of up to $70 \%$. In all simulated experiments, only marginal differences between the obtained transfer efficiencies for the four cases $\mathrm{C} 1-\mathrm{C} 4$ are observed for all spin pairs. Time-dependent modulations of the rf amplitude and phase due to the radial $\mathrm{rf}$ inhomogeneity, therefore, do not seem to have a significant effect on the magnetization build up on the destination spin. On the source spin, some magnetization is lost when rf phase modulations are present (C3 and $\mathrm{C} 4$ ), which is also observed for one-spin spin lock simulations. As the radial contributions to the rf inhomogeneity are weaker in the 1.9 and $1.3 \mathrm{~mm}$ probes, similar results would be expected for these probes. Overall, these simulation results suggest that the effect of the radial inhomogeneity on $\mathrm{CP}$ polarization transfers is negligible. Only the static rf amplitude offset over the relevant sample space is important due to the volume selectivity it causes. 

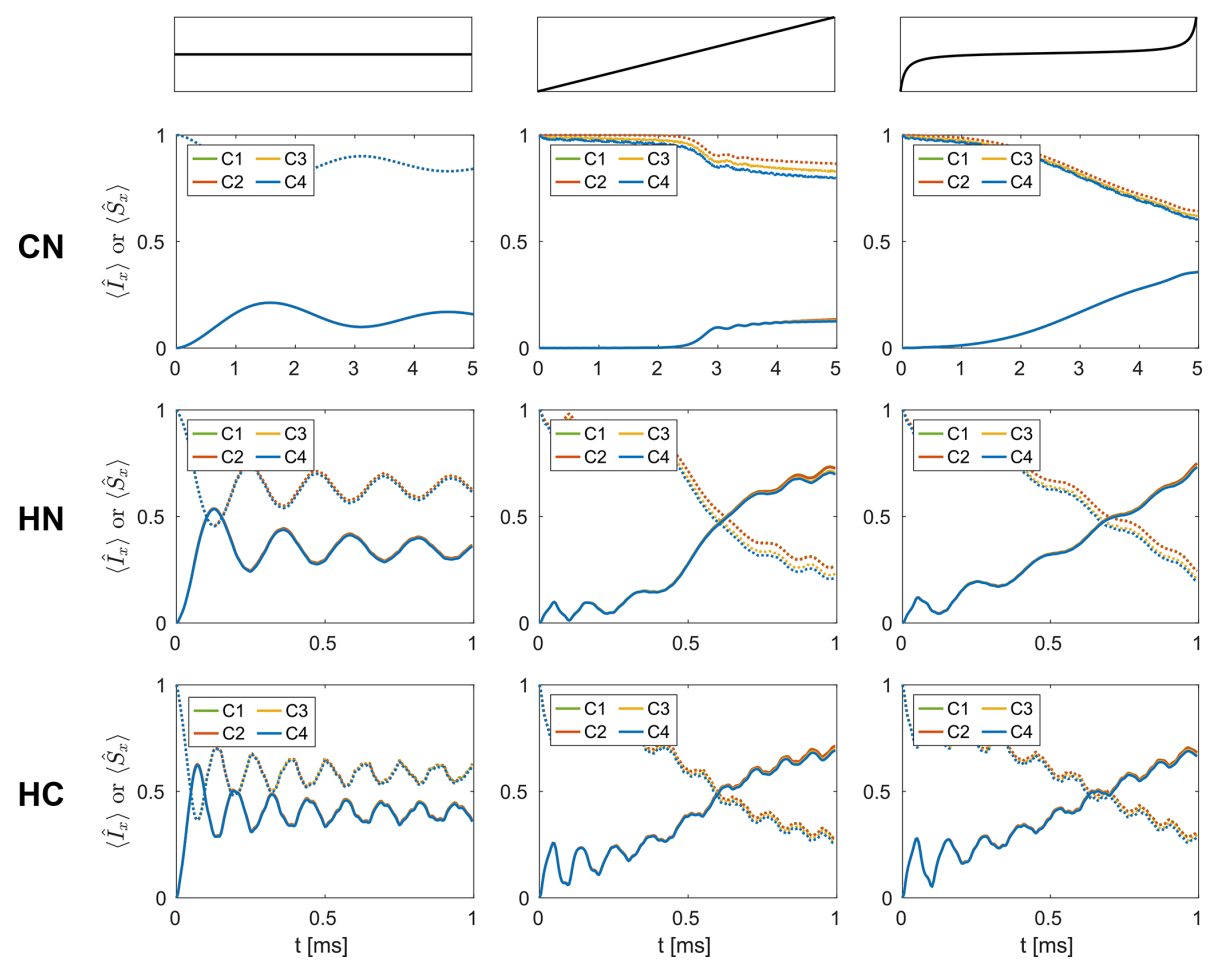

Figure 6. Simulated time evolution of the expectation value of the $\hat{I}_{x}$ (dotted lines) and $\hat{S}_{x}$ (solid lines) operators during CP zero quantum $\hat{I}_{x} \rightarrow \hat{S}_{x}$ polarization transfers in CN (top row), HN (middle row), and HC (bottom row) spin pairs for C1-C4 (see Table 1) in a $3.2 \mathrm{~mm}$ probe at a proton resonance frequency of $600 \mathrm{MHz}$, assuming a MAS frequency of $20 \mathrm{kHz}$. Shown are magnetization transfers for constant rf amplitude (left-hand column), a linear rf amplitude modulation (middle column), and a tangential rf amplitude modulation on the source spin (right-hand column). The rf amplitude on the destination spin was kept constant $(65 \mathrm{kHz}$ on N in $\mathrm{CN}, 50 \mathrm{kHz}$ on $\mathrm{N}$ in $\mathrm{HN}$, and $70 \mathrm{kHz}$ on $\mathrm{C}$ in $\mathrm{HC}$ ), and modulations of the rf amplitude on the source spin are shown in Fig. S4. Overall, no significant effects of the time-dependent amplitude and phase modulations due to radial contributions to the rf inhomogeneity are observed for the destination spin in any of the simulated transfers.

Moreover, polarization transfers in NCA and NCO twospin systems using the tm-SPICE sequences (Tošner et al., 2018) were simulated. These pulse schemes were developed using optimal control (OC) strategies, taking into account the MAS modulations of the rf field due to the radial rf inhomogeneity. The resulting magnetization transfers are shown in Fig. 7 for the $3.2 \mathrm{~mm}$ MAS probe at a proton resonance frequency of $400 \mathrm{MHz}$. Nominal rf amplitudes on both channels were set to $40 \mathrm{kHz}$, and a spinning speed of $20 \mathrm{kHz}$ was assumed. The shape files for the pulse sequences contain 1750 points, with a time resolution of $2 \mu \mathrm{s}$, corresponding to a contact time of $3.5 \mathrm{~ms}$. The time resolution for the propagation was set to $250 \mathrm{~ns}$, and the expectation value of the $\hat{I}_{x}$ and $\hat{S}_{x}$ operators was detected every $5 \mu$ s. Powder averaging was performed over 1154 crystallite orientations. Spin system parameters ( $J$ couplings, chemical shift anisotropy, CSA, and dipolar coupling tensors) were taken from Tošner et al. (2018) and can be found in Tables S2 and S3. Impressive transfer efficiencies of around $60 \%$ are obtained for both NCA (Fig. 7a) and NCO (Fig. 7b) spin pairs. However, only minor differences between the four cases $\mathrm{C} 1-\mathrm{C} 4$ are observed in these simulations. This reflects the fact that the optimization of this sequence took rf amplitude and phase modulations of different magnitude, as well as varying initial phases of these modulations, into account. Therefore, the sequence performs well under all possible conditions encountered in the rotor, leading to an increase in the robustness of the resulting sequences towards static and time-dependent rf inhomogeneity. The broad range of considered conditions stabilizes the optimization towards a broader minimum that gives a better transfer over the complete rotor.

\subsection{Rotational-echo double resonance}

In REDOR recoupling (Gullion and Schaefer, 1989a, b), the heteronuclear dipolar coupling is reintroduced by trains of two rotor-synchronized $\pi$ pulses per rotor cycle. This technique allows the quantitative measurement of dipolar couplings in heteronuclear spin pairs and has become a valuable tool in the characterization of structure (Hong, 2006; Rienstra et al., 2002; Michal and Jelinski, 1997; Jia et al., 2015) and dynamics (Schanda et al., 2010).

Numerical simulations of REDOR recoupling were performed for $\mathrm{CN}$ and $\mathrm{HN}$ spin pairs at a proton resonance fre- 
(a)

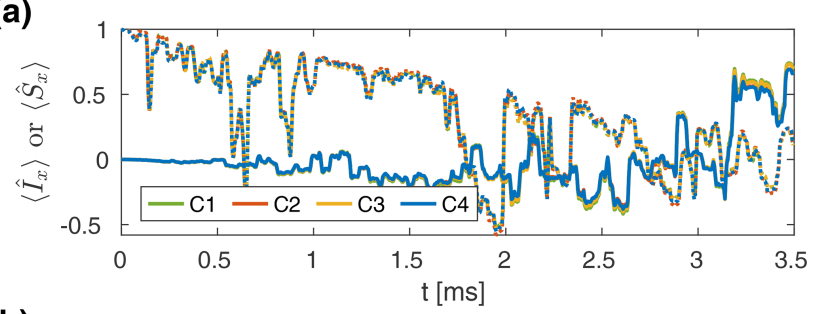

(b)

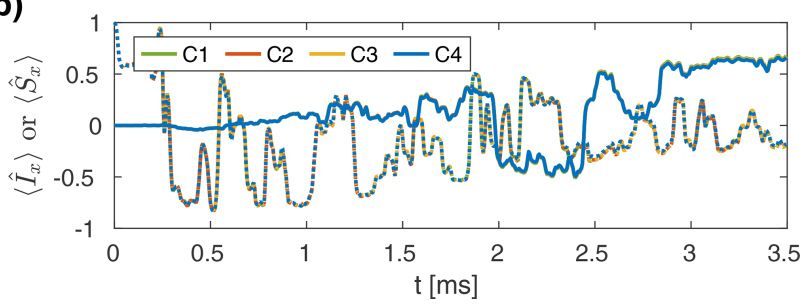

Figure 7. Simulated time evolution of the $\hat{I}_{x}$ (dotted lines; ${ }^{15} \mathrm{~N}$ ) and $\hat{S}_{x}$ (solid lines; ${ }^{13} \mathrm{C}$ ) operators during tm-SPICE (Tošner et al., 2018) CP polarization transfers in NCA (a) and NCO (b) two-spin systems (see Tables $\mathrm{S} 2$ and $\mathrm{S} 3$ for spin system parameters) in a $3.2 \mathrm{~mm}$ MAS probe at a proton resonance frequency of $400 \mathrm{MHz}$. A spinning frequency of $20 \mathrm{kHz}$ was assumed, and the nominal rf amplitudes were set to $40 \mathrm{kHz}$ on both channels. Impressive transfer efficiencies of approximately $60 \%$ are achieved in both spin pairs. However, no significant differences between the four cases $\mathrm{C} 1-\mathrm{C} 4$ are observed.

quency of $600 \mathrm{MHz}$, with an XY-4 phase cycling scheme (Gullion et al., 1990) as it is commonly implemented to generate a pure Ising-type Hamiltonian. Dipolar couplings were estimated from average bond lengths, and the anisotropy of the coupling $\frac{\delta_{\mathrm{IS}}}{2 \pi}$ was set to 2 and $24 \mathrm{kHz}$ in $\mathrm{CN}$ and $\mathrm{HN}$, respectively. Chemical shift tensors and scalar $J$ couplings were set to zero. Resulting simulated REDOR curves for CN spin pairs are shown in Fig. 8 for a $1.3 \mathrm{~mm}$ (Fig. 8a) and a $3.2 \mathrm{~mm}$ (Fig. 8b) probe, assuming a spinning frequency of $20 \mathrm{kHz}$. The nominal rf field strengths were set to $100 \mathrm{kHz}$ $(62.5 \mathrm{kHz})$ on $\mathrm{C}$ and $65 \mathrm{kHz}(50 \mathrm{kHz})$ on $\mathrm{N}$ in the $1.3 \mathrm{~mm}$ $(3.2 \mathrm{~mm})$ probe. A time resolution of $250 \mathrm{~ns}$ was chosen for the propagation, and 538 crystallite orientations were used for the powder averaging. Compared to the theoretical REDOR curve (dashed line; analytical expression including finite-pulse effects can be found in Jaroniec et al., 2000), considerably lower recoupling efficiencies are obtained for $\mathrm{C} 1-$ C4 in both probes (Nishimura et al., 2001). However, only minor differences between the four cases are observed with amplitude modulations ( $\mathrm{C} 2$ and $\mathrm{C} 4)$, leading to a slight deterioration of the recoupling performance. These effects are very similar for both probes. The relative timing of the rotorsynchronized $\pi$ pulses in the REDOR sequence with respect to the time-dependent rf field amplitude and phase modulations only has a marginal effect on the recoupling performance (see Fig. S6 for further details). Simulated REDOR curves for the $\mathrm{HN}$ spin system in the $1.9 \mathrm{~mm}$ probe, assuming a spinning frequency of $40 \mathrm{kHz}$, are shown in Fig. $8 \mathrm{c}$ and d. As the dipolar coupling in the HN spin pair is too large to allow sufficient sampling of the REDOR curve, modified REDOR implementations were simulated in which one (Gullion and Schaefer, 1989b, Fig. 8c) or both (Jain et al., 2019, Fig. 8d) of the pulses in the basic building block are shifted. The corresponding pulse sequences are shown in Fig. S5. These schemes lead to a scaling of the effective dipolar coupling and, thus, allow sufficient sampling of the REDOR curve even for strongly dipolar-coupled spin pairs. A time resolution of $125 \mathrm{~ns}$ was chosen, and the nominal rf fields were set to $125 \mathrm{kHz}$ on $\mathrm{H}$ and $50 \mathrm{kHz}$ on $\mathrm{N}$. Powder averaging was performed for 10000 crystallite orientations. Theoretical REDOR curves are again indicated by the dashed lines (analytical expressions, including finite-pulse effects, can be found in Schanda et al., 2011, for Fig. 8c and Jain et al., 2019, for Fig. 8d). For both shifting regimes, only slight deviations from the theoretical curves are observed. Moreover, resulting REDOR curves for $\mathrm{C} 1-\mathrm{C} 4$ are identical, indicating that timedependent amplitude and phase modulations have no effect on the recoupling performance in strongly dipolar-coupled spin pairs. Overall, the REDOR sequence seems to be predominantly affected by the static rf inhomogeneity which causes deviations in the pulse flip angles from the desired $180^{\circ}$ due to average rf field amplitude deviations.

\subsection{Symmetry-based $\mathrm{CN}$ recoupling $-\mathrm{C} 7$ and POST-C7}

Symmetry-based $\mathrm{C} N_{\kappa}^{v}$ sequences represent an important class of homonuclear recoupling sequences. Since the first introduction of the original $\mathrm{C} 72 \frac{1}{2}$ sequence (Lee et al., 1995), many other symmetry-based sequences have been proposed and characterized; however, only the $\mathrm{C}_{2}^{1}$ and the POSTC7 sequence (Hohwy et al., 1998), where the basic $\mathrm{C}_{\phi}=$ $(2 \pi)_{\phi}(2 \pi)_{\phi+\pi}$ is replaced by the cyclically permuted $\mathrm{C}_{\phi}=$ $\left(\frac{\pi}{2}\right)_{\phi}(2 \pi)_{\phi+\pi}\left(\frac{3 \pi}{2}\right)_{\phi}$ POST element, were considered in this work.

Numerical simulations of $\hat{S}_{1 z} \rightarrow \hat{S}_{2 z}$ polarization transfers during C7 and POST-C7 recoupling were performed for $\mathrm{CC}$ two-spin systems under conditions typical for a $3.2 \mathrm{~mm}$ MAS probe at a carbon resonance frequency of $150 \mathrm{MHz}$. The nominal rf field amplitude was set to $70 \mathrm{kHz}$, and a spinning frequency of $10 \mathrm{kHz}$ assumed. A time resolution of approximately $714 \mathrm{~ns}$ was chosen, and 538 crystallite orientations were used for the powder averaging. The time evolution of the expectation values of $\hat{S}_{1 z}$ and $\hat{S}_{2 z}$ in a CC spin pair with isotropic chemical shifts that are symmetric around zero $\left(\Omega_{1}=-\Omega_{2}\right)$ is shown in Fig. 9 for C7 (Fig. 9a) and POST-C7 (Fig. 9b) for the cases C1-C4. The anisotropy of the dipolar coupling tensor was estimated from average bond lengths and $\frac{\delta_{\mathrm{IS}}}{2 \pi}$ set to $4.5 \mathrm{kHz}$. For both sequences, transfer efficiencies of approximately $70 \%$ are achieved in the first transient for a mixing time of approximately $10 \mathrm{~ms}$. Amplitude modulations due to the radial rf field inhomogeneity ( $\mathrm{C} 2$ and $\mathrm{C} 4$ ) lead to a slight deterioration in the recoupling performance. This effect is more pronounced for $\mathrm{C} 7$, indicating 

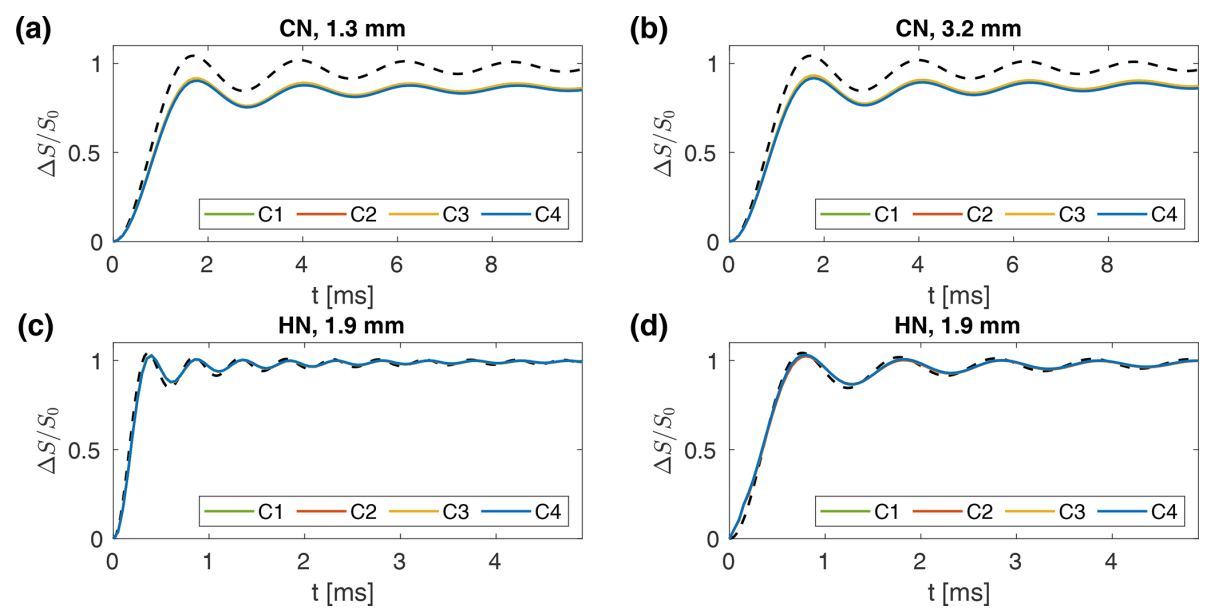

Figure 8. (a, b) Simulated REDOR curves for a CN spin pair, with a value of $2 \mathrm{kHz}$ for the anisotropy of the dipolar coupling tensor $\frac{\delta_{\mathrm{IS}}}{2 \pi}$ at a carbon resonance frequency of $150 \mathrm{MHz}$ in a $1.3 \mathrm{~mm}$ MAS probe (a) and a $3.2 \mathrm{~mm}$ MAS probe (b), assuming a spinning frequency of $20 \mathrm{kHz}$. The nominal rf field strengths were set to $100 \mathrm{kHz}(62.5 \mathrm{kHz})$ for $\mathrm{C}$ and $62.5 \mathrm{kHz}(50 \mathrm{kHz})$ for $\mathrm{N}$ in the $1.3 \mathrm{~mm}(3.2 \mathrm{~mm})$ probe. For all four cases (C1-C4), the resulting recoupling efficiencies are significantly lower than the theoretical REDOR curve (dashed line). Amplitude modulations (C2 and $\mathrm{C} 4)$ lead to a further marginal deterioration of the recoupling efficiency. The remaining two cases (C1 and C3) are indistinguishable. (c, d) Simulated REDOR curves for a $\mathrm{HN}$ spin pair with a $\frac{\delta_{\mathrm{IS}}}{2 \pi}$ of $24 \mathrm{kHz}$ at a proton resonance frequency of $600 \mathrm{MHz}$ in a $1.9 \mathrm{~mm}$ MAS probe, assuming a spinning frequency of $40 \mathrm{kHz}$. The nominal rf field strengths were set to $125 \mathrm{kHz}$ for $\mathrm{H}$ and $50 \mathrm{kHz}$ for $\mathrm{N}$. A scaling of the effective dipolar coupling is achieved by shifting one pulse (c; delay until first pulse $\left.t_{1}=2.5 \mu \mathrm{s}\right)$ or both pulses (d) delay until first pulse $t_{1}=16 \mu \mathrm{s}$ ) per rotor period. No significant deviation in any of the four cases (C1-C4) from the theoretical REDOR curves (dashed lines) is observed, indicating the robustness of these REDOR implementations towards rf inhomogeneity.

the improved robustness of the POST-C7 sequence. At longer times, a loss of magnetization on both spins is observed when time-dependent amplitude modulations are taken into account. Interestingly, the overall order of the cases is different for the two sequences. For $\mathrm{C} 7$ recoupling, higher transfer efficiencies are observed for $\mathrm{C} 4$ in comparison to $\mathrm{C} 2$, whereas this order is reversed for POST-C7. Thus POST-C7 seems to be more sensitive to combined amplitude and phase modulations (C4). Figure 9c (C7) and d (POST-C7) show simulation results of the same spin system for a spatially restricted sample (central third along the rotor axis). This restriction of the sample space mitigates the effects of amplitude modulations, and only very marginal differences between the four cases are observed for both sequences. In order to further investigate the robustness of the two sequences, simulations in a second model system with a large CSA tensor were performed at a lower external magnetic field $(75 \mathrm{MHz}$ carbon resonance frequency). The parameters of this spin system were based on phthalic acid (Hellwagner et al., 2017, see Table S4). Significantly lower overall transfer efficiencies (approximately $50 \%$ ) are observed (see Fig. 9e and f), and for both sequences, rf field amplitude modulations (C2 and $\mathrm{C} 4)$ further deteriorate the recoupling efficiency. This decrease is less pronounced for POST-C7 (see Fig. 9f), again indicating its improved robustness. We have not carried out a complete Floquet analysis of the effects caused by the time-dependent rf field amplitudes. We believe that the decreased efficiency is due to the appearance of effective fields that shift the res- onance condition slightly as is the case for pulse transients (Hellwagner et al., 2017).

\subsection{Frequency-switched Lee-Goldburg decoupling}

\subsubsection{Numerical simulation and experimental results}

Frequency-switched Lee-Goldburg (FSLG) decoupling is a homonuclear dipolar decoupling technique that can be used in combination with MAS to improve resolution of spectra for dipolar-coupled homonuclear spin systems (Lee and Goldburg, 1965; Goldburg and Lee, 1963; Bielecki et al., 1989, 1990). The experiment is based on off-resonance rf irradiation, leading to a truncation of the second-rank spin tensor of the homonuclear dipolar coupling by an effective radio-frequency field inclined at an angle $\theta_{\mathrm{m}} \approx 54.74^{\circ}$ with respect to the static magnetic field. Experimentally, FSLG can also be implemented using on-resonance irradiation with a constant rf field amplitude and a continuous phase ramp to generate the frequency offset. The total cycle time is divided into two intervals of equal length during which the phase is rotated in opposite directions (inverting the offset) and with a phase jump of $180^{\circ}$ in between.

The effects of the radial part of the rf field inhomogeneity on the residual linewidth under FSLG decoupling were simulated for a homonuclear dipolar-coupled three-spin system in a $3.2 \mathrm{~mm}$ MAS probe at a proton resonance frequency of $600 \mathrm{MHz}$, assuming a MAS frequency of $12.5 \mathrm{kHz}$. The nominal rf field amplitude was set to $102.06 \mathrm{kHz}$, corre- 
(a)

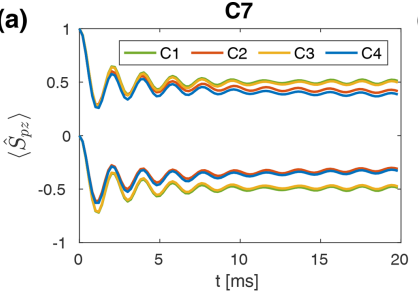

(c)

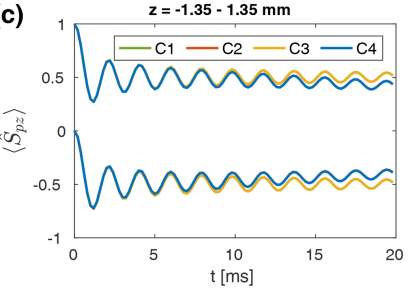

(e)

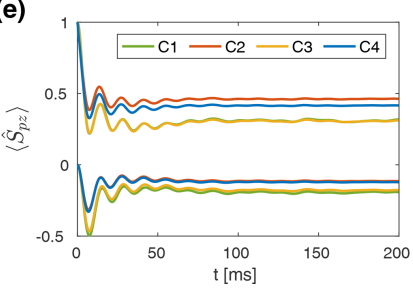

(b)

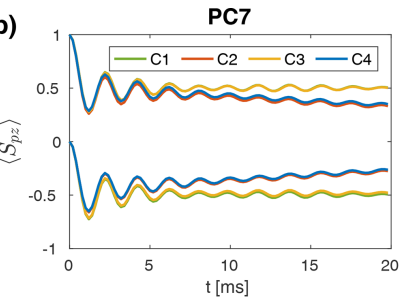

(d)

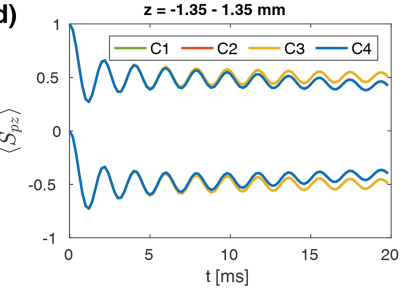

(f)

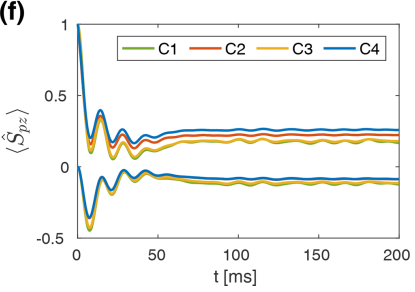

Figure 9. Simulated $\hat{S}_{1 z} \rightarrow \hat{S}_{2 z}$ polarization transfers in CC spin systems during C7 (a, c, e) and POST-C7 (b, d, f) recoupling for $\mathrm{C} 1-\mathrm{C} 4$ in a $3.2 \mathrm{~mm}$ probe. A nominal rf field amplitude of $70 \mathrm{kHz}$ and a spinning frequency of $10 \mathrm{kHz}$ was assumed. Simulations shown in panels (a-d) were performed at a carbon resonance frequency of $150 \mathrm{MHz}$. Resulting expectation values of $\hat{S}_{1 z}$ and $\hat{S}_{2 z}$ for a spin pair with isotropic chemical shifts that are symmetric around zero $\left(\Omega_{1}=-\Omega_{2}\right)$ and a value of $4.5 \mathrm{kHz}$ for $\frac{\delta_{\mathrm{IS}}}{2 \pi}$ are shown for the full rotor (a, b) and for a spatially restricted sample (central third along rotor axis; (c, d). Overall, rf field amplitude modulations (C2 and $\mathrm{C} 4$ ) lead to a slight deterioration in the recoupling performance for both sequences. The other two cases $(\mathrm{C} 1$ and $\mathrm{C} 3)$ are nearly identical. (e, f) Simulation results for a $\mathrm{CC}$ spin pair with considerable CSA and a value of $-585 \mathrm{~Hz}$ for $\frac{\delta_{\mathrm{IS}}}{2 \pi}$ at a lower magnetic field (carbon resonance frequency of $75 \mathrm{MHz}$ ) for C7 (e) and POSTC7 (f) recoupling. Lower overall transfer efficiencies are observed for this spin system, and modulations of the rf field amplitude (C2 and $\mathrm{C} 4$ ) further deteriorate the recoupling performance (C1 and $\mathrm{C} 3$ are indistinguishable). This effect is stronger for C7.

sponding to a tilting along the magic angle of an effective field with a strength of $125 \mathrm{kHz}$ (FSLG cycle time of $16 \mu \mathrm{s}$ ). Using such a synchronization between the FSLG sequence and the sample spinning makes the simulations much more efficient than an asynchronous implementation, while at the same time avoiding all resonance conditions up to and including the second order. The FSLG decoupling was implemented using a phase ramp with a time resolution of $50 \mathrm{~ns}$. The same time resolution was chosen for the propagation of the Hamiltonian. The initial density operator was set to $\hat{F}_{y}=\sum_{p=1}^{3} \hat{I}_{p y}$ and transverse magnetization components detected every $48 \mu$ s (three FSLG cycles). A total of 8192 data

points were acquired, and the free induction decay (FID) processed in MATLAB. Powder averaging was performed over 1154 orientations. The parameters characterizing the chemical shift and dipolar coupling tensors were chosen to mimic a $\mathrm{CH}_{2}$ group, with couplings to an additional remote spin, and can be found in Tables S5 and S6. Scalar $J$ couplings were neglected and set to zero.

Simulated spectra of the three-spin system are shown in Fig. 10a for $\mathrm{C} 1-\mathrm{C} 4$. In all four cases, all resonances show strong asymmetric features on the left-hand side of the spectral line due to the distribution of the chemical shift scaling factors (Hellwagner et al., 2020). Considerable additional line broadening is observed when amplitude modulations are taken into account (C2 and C4). As the same linewidths were obtained in simulations of an asynchronous implementation of FSLG decoupling (MAS frequency of approximately $14.1 \mathrm{kHz}$; see Fig. S7), the broadening is not caused by resonance effects. This effect is observed for all three resonances but is most pronounced for the $\mathrm{CH}_{2}$ resonance around $1.25 \mathrm{kHz}$. The additional time dependence of the rf phase in $\mathrm{C} 4$ results in no additional broadening, and the two remaining cases $(\mathrm{C} 1$ and $\mathrm{C} 3)$ are indistinguishable. Phase modulation, therefore, does not seem to have an influence on the obtained linewidth. Figure 10 also shows simulated FSLG spectra for radial slices of the simulated $r z$ plane at $r=0.65$ (Fig. 10b) and $1.3 \mathrm{~mm}$ (Fig. 10c) and for a spatially restricted sample space (central third along the rotor axis; $z=-1.35-1.35 \mathrm{~mm}$; all $r$ values; Fig. 10d). As the magnitude of the rf amplitude modulations increases towards the rotor edges (see Fig. 2), significantly stronger broadening is observed for radial slices closer to the coil windings. Spatial restriction of the sample space to the central third leads to a reduction in the linewidth. This line narrowing is significantly more pronounced for the resonance at $-2.75 \mathrm{kHz}$, where the foot on the left-hand side of the resonance is eliminated for all four cases. For C2 and C4, broadening is observed even in this spatially restricted sample, indicating that time-dependent amplitude modulations without a static rf amplitude offset still result in contributions to the residual linewidth. Simulations were also performed for a six-spin system, and qualitatively similar results were obtained.

In order to observe this broadening experimentally, the sample space has to be restricted to areas close to the coil windings where strong rf field amplitude modulations occur. This could, in principle, be achieved by physically restricting the sample using cylindrical spacers. However, homogeneous packing in such a sample is difficult to achieve. Alternatively, nutation-frequency-selective pulses, as described in Aebischer et al. (2020), can be used to select the desired areas which also correspond to high average rf field amplitudes. Figure 11 shows the FSLG decoupled proton spectra of L-histidine measured at a proton resonance frequency of $500 \mathrm{MHz}$ in a Bruker $1.9 \mathrm{~mm}$ MAS probe, using a $2 \mathrm{~ms}$ I-BURP-2 pulse in the spin lock frame for the $B_{1}$ field selection of areas where the rf field amplitude corre- 

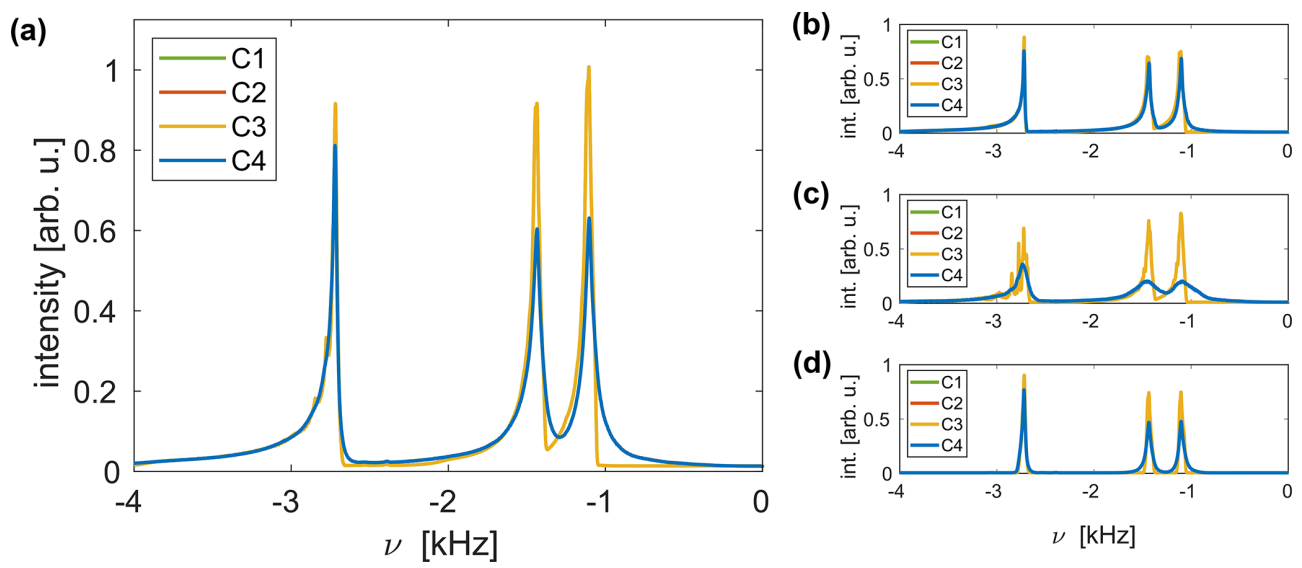

Figure 10. Simulated FSLG decoupled proton spectra of a three-spin system at a resonance frequency of $600 \mathrm{MHz}$ for a $3.2 \mathrm{~mm}$ MAS probe, assuming a MAS frequency of $12.5 \mathrm{kHz}$ for $\mathrm{C} 1-\mathrm{C} 4$. The rf field strength along the magic angle was set to $125 \mathrm{kHz}$. (a) Spectrum for the full $r z$ plane. Significant additional line broadening is observed when amplitude modulations are taken into account (C2 and C4). The two remaining cases are indistinguishable, indicating that rf phase modulations do not contribute to the residual linewidth. (b, $\mathbf{c}, \mathbf{d})$ Contributions from radial slices (all $z$ values for given $r$ ) at $r=0.65$ (b) and $1.3 \mathrm{~mm}(\mathbf{c})$, as well as the spectrum of a spatially restricted sample (central third along the rotor axis; $z=-1.35-1.35 \mathrm{~mm}$; all $r$ values; $\mathbf{d}$ ). Stronger line broadening is observed towards the edges of the rotor where rf modulations are more pronounced. Significantly narrower lines are obtained for the restricted sample; however, considerable broadening is still observed when time-dependent rf amplitude modulations are taken into account (C2 and $\mathrm{C} 4)$. The observed splitting of the line for $\mathrm{C} 1$ and $\mathrm{C} 3$ in (c) can be attributed to the distribution of the isotropic chemical shift scaling factors in this radial slice (see Fig. S8 for details).

sponds to the nominal value (see Fig. S9 for a simulated inversion profile). Spectra were recorded with different $B_{1}$ field strengths for the FSLG decoupling at spinning frequencies of 14 and $28 \mathrm{kHz}$. No significant improvement in the obtained linewidth is observed for higher MAS frequencies and stronger decoupling field strengths. This indicates that the residual linewidth in these spectra is not decoupling limited which, therefore, prohibits the experimental characterization of the additional broadening caused by rf field amplitude modulations due to the radial rf field inhomogeneity.

\subsubsection{Floquet analysis}

In order to gain physical insight into the origin of the observed line broadening in FSLG-decoupled spectra due to rf field amplitude modulations, scaling factors for the first- and second-order contributions to the effective Hamiltonian were computed (see Sect. 3.1.1 for more details). As a simple measure for the magnitude of contributing first-order terms the norms of one-spin coefficients,

$\bar{a}_{\chi}^{(k)}=\sqrt{\sum_{\chi^{\prime}} \sum_{\ell=-1}^{1} a_{\chi^{\prime}}^{(k, \ell)^{2}}}$,

and two-spin coefficients,

$\bar{a}_{\chi \mu}^{(k)}=\sqrt{\sum_{\chi^{\prime}, \mu^{\prime}} \sum_{\ell=-2}^{2} a_{\chi^{\prime} \mu^{\prime}}^{(k, \ell)^{2}},}$

were computed.
Interaction frame trajectories using the rf field distribution in a $3.2 \mathrm{~mm}$ MAS probe during FSLG decoupling were computed numerically in MATLAB with a time resolution of $50 \mathrm{~ns}$ and the Fourier coefficients extracted. The spinning frequency was chosen to be $12.5 \mathrm{kHz}$ and the effective field strength along the magic angle set to $125 \mathrm{kHz}$, corresponding to a modulation frequency of the rf Hamiltonian of $62.5 \mathrm{kHz}$. This leads to the synchronization of the MAS rotation and the rf irradiation after a single rotor cycle or five FSLG cycles. This choice of frequencies should avoid all resonance conditions up to and including the second order. Relative rf field amplitude and phase modulations were modelled as Fourier series, and fitted Fourier coefficients up to the fourth order were used as input (see Sect. 2). In analogy to the treatment of the rf field inhomogeneity in numerical simulations, amplitude and phase modulations were considered separately, and the four cases $\mathrm{C} 1-\mathrm{C} 4$ summarized in Table 1 studied.

A full FSLG cycle assuming a time-independent rf Hamiltonian and an ideal phase ramp with a $180^{\circ}$ phase shift in the middle consists of two $\beta$ rotations with an opposite direction about the effective field. The overall propagator would, thus, be the unity operator and $\omega_{\text {eff }}=0$ in Eq. (12). However, the time-dependent modulations of the rf amplitude and phase due to the radial rf inhomogeneity can give rise to an additional effective field. The magnitude of this field as a function of the position within the sample space in the $3.2 \mathrm{~mm}$ probe is shown in Fig. 12. As the static rf field inhomogeneity does not lead to additional effective fields, only cases in which either the rf field amplitude, the phase, or both are time dependent (C2, C3 and C4) are shown. Effective fields arise 

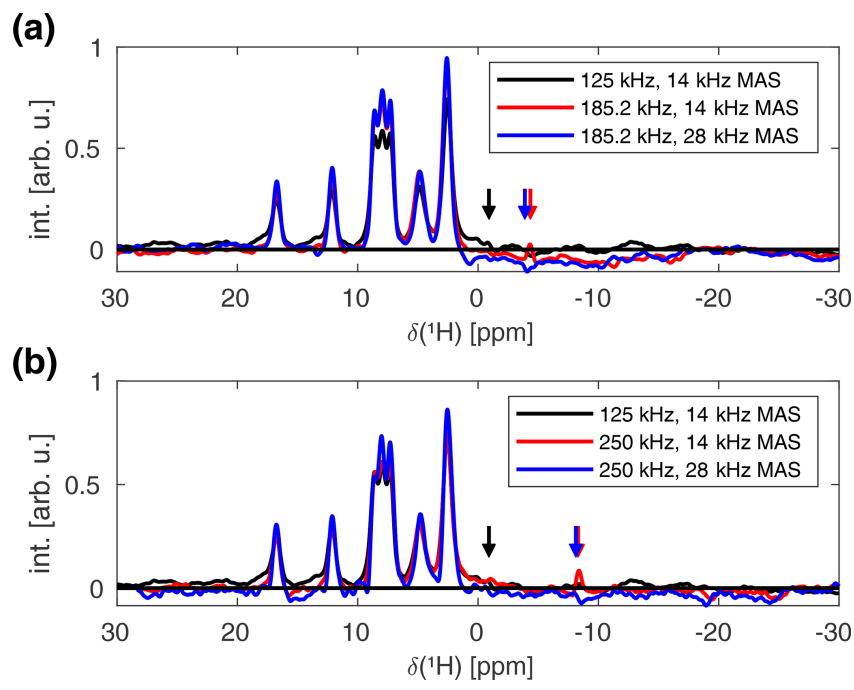

Figure 11. Experimental FSLG decoupled proton spectra of natural-abundance L-histidine recorded at a proton resonance frequency of $500 \mathrm{MHz}$, using a $1.9 \mathrm{~mm}$ Bruker MAS probe. All spectra were recorded using a $2 \mathrm{~ms}$ I-BURP-2 pulse with a modulation frequency of $100 \mathrm{kHz}$ (corresponding to the nominal rf field amplitude as determined using a nutation spectrum) for the $B_{1}$ selection (Aebischer et al., 2020). The effective rf field strength along the magic angle during the FSLG decoupling was set to 125 (a, b), 185.2 (a), and $250 \mathrm{kHz}$ (b) at MAS frequencies of 14 and $28 \mathrm{kHz}$. The arrows indicate the positions of the carrier frequency. No significant improvements are observed for stronger $B_{1}$ fields and higher spinning frequencies, indicating that the residual linewidth is not decoupling limited.

mainly at the edges of the rotor (large $r$ and $z$ ) where modulations are strongest. Amplitude and phase modulations alone (C2 and C3 respectively) lead to very small effective fields (max. $50 \mathrm{~Hz}$ ), whereas larger effective fields (up to $400 \mathrm{~Hz}$ ) result for combined amplitude and phase modulations (C4). In comparison to the rotor frequency and the basic modulation frequency of the FSLG sequence, these additional fields are small and will most likely not have any significant effects, except for a small change in the effective field direction and magnitude.

In the first-order approximation, the relevant scaling factors are those of the chemical shift $\left(a_{x}^{(k, \ell)}\right.$ with $k=$ $0, \pm 1, \pm 2)$ and those of the dipolar coupling $\left(a_{\chi \mu}^{(k, \ell)}\right.$ with $k= \pm 1, \pm 2$ ). These can contribute to the first-order effective Hamiltonian (see Eq. 27), since the modulation by the rf field amplitude can be compensated by the time dependence due to MAS. The resulting norm of the $a_{\chi}^{(k, \ell)}$ coefficients (Eq. 38) as a function of the position within the sample space is shown in Fig. 13a for C1-C4. As coefficients are symmetric $\left(\bar{a}_{\chi}^{(k)}=\bar{a}_{\chi}^{(-k)}\right)$, only those corresponding to $k=0,1$ and 2 are shown. The scaling of the isotropic chemical shift $(k=0)$ is close to the ideal value of $\cos \left(\theta_{\mathrm{m}}\right) \approx 0.577$ in regions of the rotor where the rf field amplitude is comparable to the nominal value. Towards the edges of the rotor, the rf field

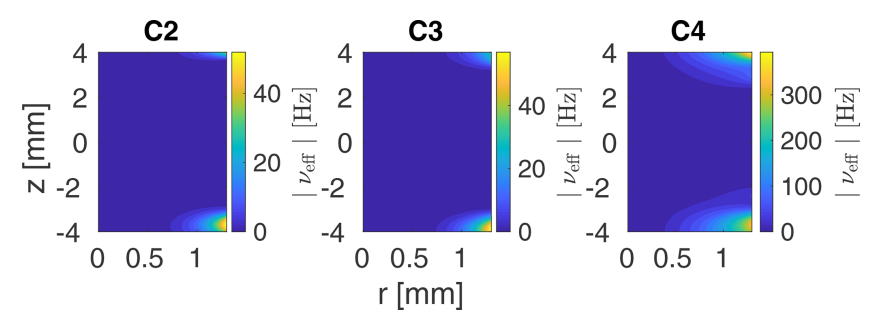

Figure 12. Effective nutation frequencies $v_{\text {eff }}$ over a MAS period during FSLG decoupling as a function of the position within the $3.2 \mathrm{~mm}$ MAS probe for $\mathrm{C} 2, \mathrm{C} 3$, and C4. Effective fields were extracted from interaction frame trajectories computed for a field strength of $125 \mathrm{kHz}$ along the magic angle and a MAS frequency of $12.5 \mathrm{kHz}$. Small effective fields of up to $50 \mathrm{~Hz}$ arise for $\mathrm{rf}$ amplitude and phase modulations alone (C2 and C3). Substantially larger fields up to $400 \mathrm{~Hz}$ are observed for combined modulations (C4). In general, effective fields of considerable size are only obtained at the rotor edges, and even the maximum resulting magnitudes remain small compared to the nominal rf field strength and the rotor frequency.

amplitude decreases, leading to a smaller tilt angle of the effective field during FSLG and, thus, an increase in the scaling factor. The time-modulated part of the rf field inhomogeneity does not appear to have any influence on the isotropic chemical shift, as no significant differences between the four cases are observed. Time-dependent amplitude modulations ( $\mathrm{C} 2$ and $\mathrm{C} 4)$ lead to additional non-zero coefficients for chemical shift contributions with $k \neq 0$ that can also contribute to the first-order effective Hamiltonian for $k= \pm 1, \pm 2$ (see Eq. 27) where parts of the CSA tensor become time independent. These contributions will be strongest at the very edges of the sample space (large $r$ and $z$ ), but non-zero coefficients are also obtained in the central third of the rotor close to the coil windings.

Under ideal conditions, the FSLG decoupling scheme leads to the averaging of the anisotropic dipolar coupling in the first-order approximation, and the corresponding scaling factors would be zero. However, dipolar coupling terms are reintroduced when $\mathrm{rf}$ modulations are taken into account. The norm of the relevant $a_{\chi \mu}^{(k, \ell)}$ coefficients (Eq. 39) is shown in Fig. 13b for the $3.2 \mathrm{~mm}$ MAS probe. Again, only the $k= \pm 1, \pm 2$ terms can contribute to the first-order effective Hamiltonian and partially reintroduce Fourier components of the dipolar coupling. As was the case for the chemical shift scaling factors, the coefficients are symmetric, and thus, only those for $k=1$ and 2 are shown. Amplitude modulations ( $\mathrm{C} 2$ and $\mathrm{C} 4)$ lead to significant $k=1$ scaling factors, and non-zero coefficients are not only obtained at the very edges of the rotor but also in the central third close to the coil windings. The additional phase modulation in $\mathrm{C} 4$ does not have an influence, and amplitude modulations alone thus seem to be responsible for the reintroduction of the firstorder coupling terms. The contribution of individual $a_{\chi \mu}^{(k=1)}$ coefficients to the norm are shown in Fig. S10 for C4. Sig- 
(a)
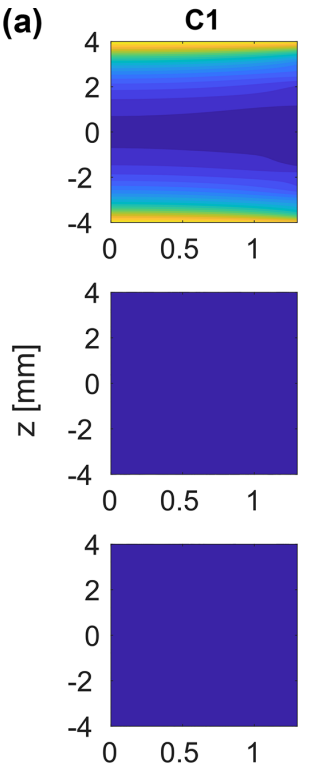

(b)
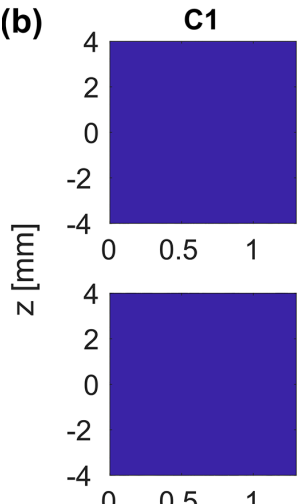

C2
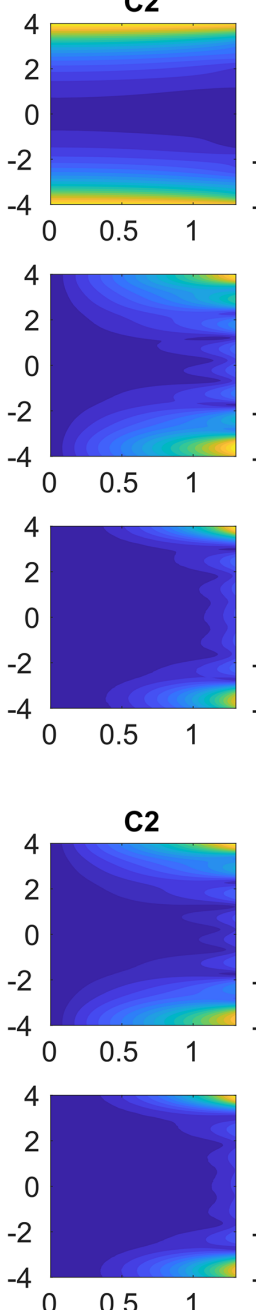
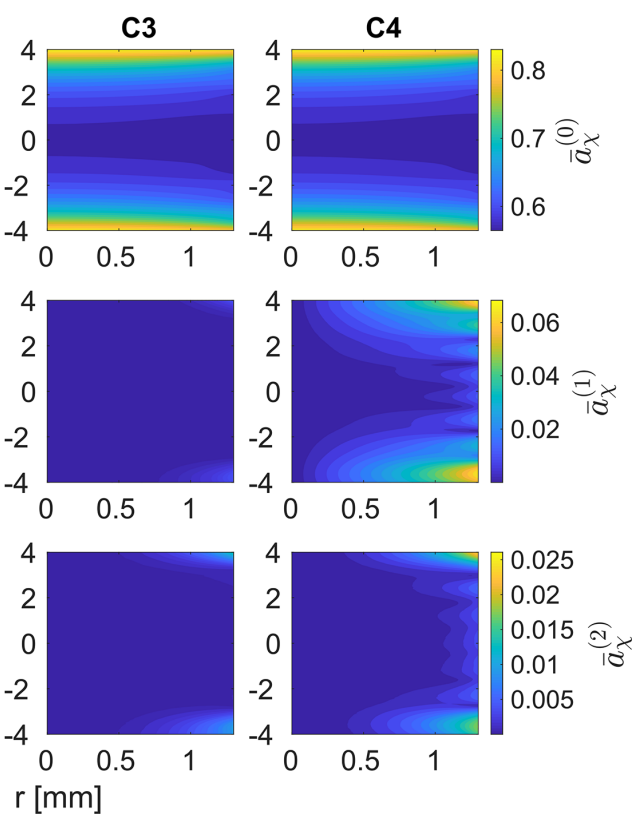

C3
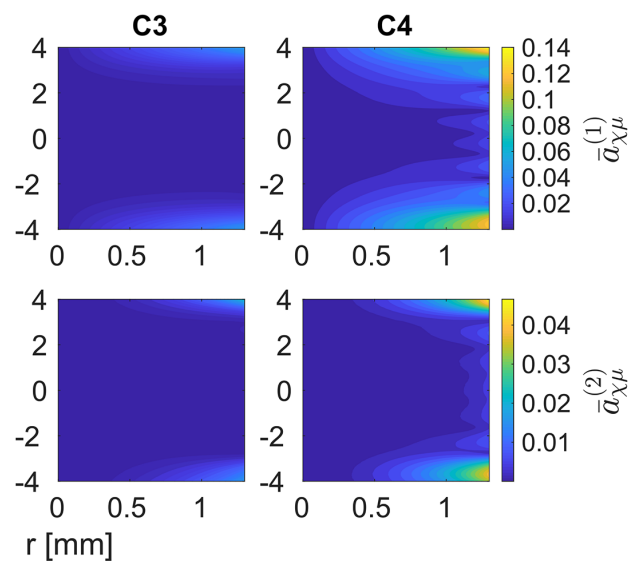

Figure 13. Norm of the scaling factors of the chemical shift terms $\bar{a}_{\chi}^{(k)}$ (for $k=0,1,2$, a) and the homonuclear dipolar coupling terms $\bar{a}_{\chi \mu}^{(k)}$ (for $k=1,2, \mathbf{b}$ ) in the first-order effective Hamiltonian for FSLG decoupling for $\mathrm{C} 1-\mathrm{C} 4$. Coefficients were extracted from interaction frame trajectories of single-spin operators for a nominal rf field strength of $125 \mathrm{kHz}$ along the magic angle and a MAS frequency of $12.5 \mathrm{kHz}$ in a $3.2 \mathrm{~mm}$ MAS probe. (a) The scaling of the isotropic chemical shift $(k=0)$ is unaffected by the time-dependent rf field modulations (no difference between $\mathrm{C} 1-\mathrm{C} 4)$ and is close to the ideal value of $\cos \theta_{\mathrm{m}} \approx 0.577$ in the centre of the rotor, where the rf field amplitude corresponds to the nominal rf field strength. Scaling factors increase towards the rotor edges where the rf field amplitude is significantly lower, leading to a smaller tilt angle. Additional non-zero coefficients for $k=1$ and 2 terms are obtained when amplitude modulations are taken into account (C2 and C4). (b) Amplitude modulations ( $\mathrm{C} 2$ and $\mathrm{C} 4$ ) lead to non-zero scaling factors and, thus, to the reintroduction of dipolar couplings in areas where strong modulations occur. No significant effects are observed for the two remaining cases.

nificant $a_{\chi \mu}^{(k=1)}$ are obtained for $\hat{I}_{p z} \hat{I}_{q x}, \hat{I}_{p x} \hat{I}_{q x}$, and $\hat{I}_{p z} \hat{I}_{q z}$ terms. These first-order time-independent homonuclear coupling terms contribute to the residual linewidth under FSLG decoupling and, thus, lead to an additional line broadening. Numerical simulations taking only the first-order effective Hamiltonian into account confirmed that the observed line broadening for $\mathrm{C} 4$ in simulated spectra (see Fig. 10) can indeed be attributed to the first-order contributions to the effective Hamiltonian (see Fig. S11).
In principle, the second-order effective Hamiltonian during FSLG decoupling contains three types of commutator cross-terms. However, contributions from chemical shift cross-terms $\left(\hat{\overline{\mathcal{H}}}_{\mathrm{I} \otimes \mathrm{I}}\right)$ only contain one-spin operators and will, thus, lead to an additional effective field and will only weakly influence the residual linewidth under FSLG by changing the direction or magnitude of the effective field. The same is true for the one-spin component of the dipolar-dipolar crossterms $\left(\hat{\mathcal{\mathcal { H }}}_{\mathrm{II} \otimes \mathrm{II}}\right)$. This leaves only two sources of coupling terms in the second-order effective Hamiltonian, namely the 
three-spin contribution of dipolar-dipolar cross-terms and commutators between chemical shift and dipolar terms. Out of these two, the former will be most relevant for the residual linewidth as dipolar couplings are generally much larger than typical chemical shifts. The corresponding $p_{\mu \chi \xi}^{\left(n_{1}, n_{2}\right)}$ scaling factors for the three-spin contribution in $\hat{\overline{\mathcal{H}}}_{\text {II } \otimes \text { II }}$ were computed according to Eq. (35). Because the effective fields generated by the modulations of the rf field amplitude and phase are small (see Fig. 12), $v=0$ was excluded from the summation in order to avoid near-resonance conditions and the norm,

$\bar{p}_{3}^{\left(n_{1}, n_{2}\right)}=\sqrt{\sum_{\mu, \chi, \xi} p_{\mu \chi \xi}^{\left(n_{1}, n_{2}\right)^{2}}}$,

was computed to characterize the strength of the three-spin coupling terms. Logarithmic contour plots for the resulting $\bar{p}_{3}^{\left(n_{1}, n_{2}\right)}$ for $\mathrm{C} 1-\mathrm{C} 4$ in the $3.2 \mathrm{~mm}$ MAS probe are shown in Fig. 14. Scaling factors are shown for $n_{1}=1$ (Fig. 14a) and $n_{1}=2$ (Fig. 14b) and all possible values of the index $n_{2}$. It can be seen that time-dependent rf modulations $(\mathrm{C} 2, \mathrm{C} 3$, and $\mathrm{C} 4)$ increase the magnitude of the second-order cross-terms significantly compared to static rf amplitude and phase offsets alone (C1). However, the observed scaling factors are still negligible compared to the magnitude of the first-order terms, and no significant effect on the linewidth would be expected. In all four cases, substantially higher scaling factors are obtained for pairs of indices where $n_{1}+n_{2}=0$. These increase strongly towards the edges of the rotor along the rotor axis, but since no difference between the cases $\mathrm{C} 1-\mathrm{C} 4$ is observed, they do not seem to be influenced by the radial part of the rf inhomogeneity.

The analysis of the scaling factors of the terms contributing to the effective Hamiltonian up to the second order suggests that the static part of the rf inhomogeneity has a significant influence on the isotropic chemical shift scaling and also leads to stronger second-order contributions. However, the overall magnitude of these second-order terms remains small compared to first-order contributions. Time-dependent rf amplitude modulations have pronounced first-order effects and lead to the reintroduction of anisotropic chemical shift and dipolar coupling terms that cause line broadening (see Fig. S11). No such effects were observed for phase modulations.

\section{Conclusion and outlook}

Magic angle spinning in combination with inhomogeneous radial $\mathrm{rf}$ fields leads to a time-dependent modulation of the rf field amplitude and phase. We have investigated the effect of these time-dependent rf fields on some common solidstate NMR pulse sequences using numerical simulations and an analytical approach based on Floquet theory. In none of the investigated building blocks used in solid-state NMR ex- (a)
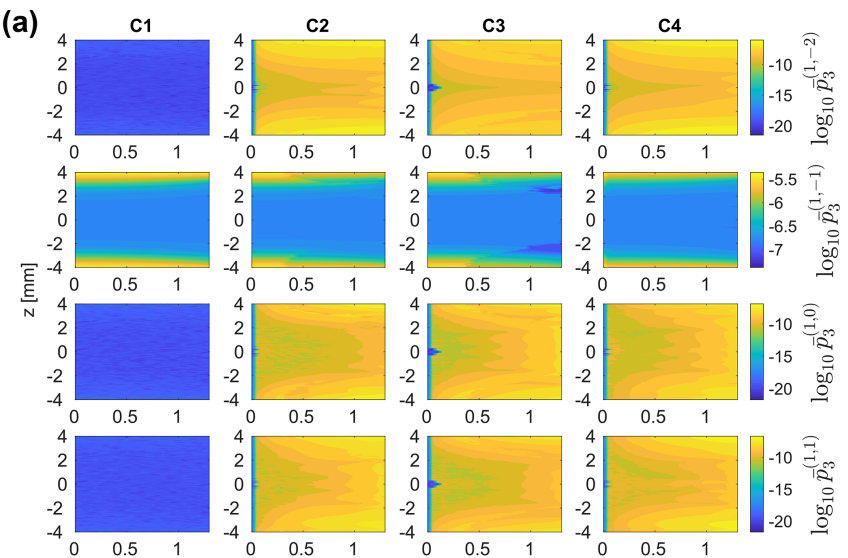

(b)
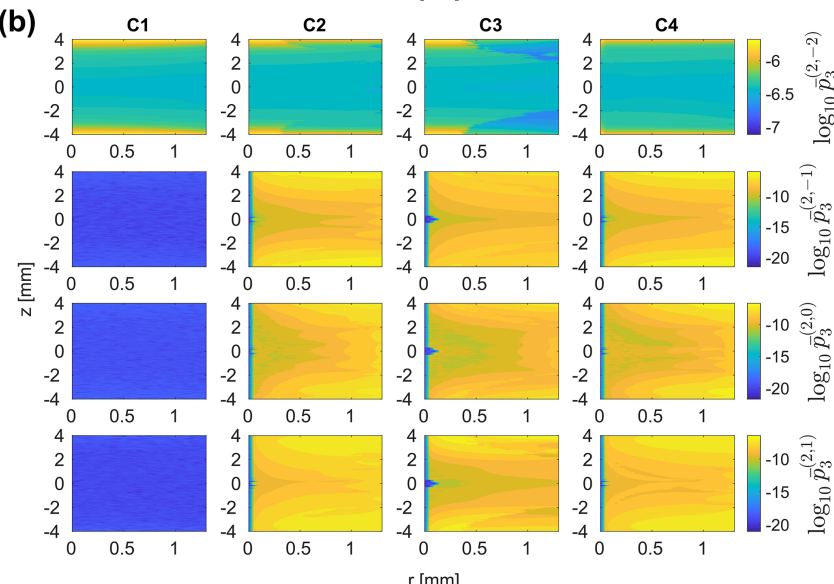

Figure 14. Magnitude of the scaling factors of the three-spin contribution to the dipolar-dipolar cross-terms in the second-order effective Hamiltonian during FSLG decoupling with an rf field strength of $125 \mathrm{kHz}$ along the magic angle at a MAS frequency of $12.5 \mathrm{kHz}$ in a $3.2 \mathrm{~mm}$ MAS probe. The resulting magnitudes are shown for $n_{1}=1$ (a) and $n_{1}=2$ (b) for all possible $n_{2}$ values with a logarithmic scale. The largest scaling factors are obtained for combinations where $n_{1}+n_{2}=0$; however, no difference between $\mathrm{C} 1-\mathrm{C} 4$ is observed. For other $n_{1}, n_{2}$ pairs, significantly larger scaling factors result when rf modulations are present $(\mathrm{C} 2, \mathrm{C} 3$, and $\mathrm{C} 4)$. Nevertheless, they remain several orders of magnitude smaller than the first-order contributions.

periments could we find significant effects from such timedependent rf fields. In nutation spectra, two distinct families of sidebands, arising due to rf field amplitude and rf field phase modulations, respectively, were observed in simulated and experimental spectra. The intensity of these sidebands can help to characterize the strength of the modulations and, thus, to give insights into the radial contribution to the rf field inhomogeneity for a given MAS probe. In the polarization transfer sequences, like Hartmann-Hahn cross-polarization, REDOR, and C7, only minor effects were observed that will most likely be of no consequence for experimental implementations. In all these sequences, the static rf field inhomo- 
geneity over the sample volume played a much larger role and leads to significant performance degradation.

In simulations of homonuclear FSLG decoupling, considerable line broadening was observed for rf field amplitude modulations. Floquet analysis of the effective Hamiltonian up to the second order revealed that this broadening is most likely due to the reintroduction of homonuclear coupling terms to the first order caused by the MAS modulation of the rf field amplitude. However, no experimental characterization of this effect was possible as the experimentally obtained linewidths were not limited by the homonuclear decoupling. Overall, the results presented in this work suggest that the influence of the MAS modulation of the rf field amplitude and phase in many pulse sequences is small and, thus, negligible for typical experimental implementations. Moreover, they manifest themselves in areas of the sample space close to the rotor edges and can, thus, be reduced by physical or virtual sample restriction. Nevertheless, these modulations can become relevant in the development of new pulse sequences based on optimal control strategies and should be taken into account in their development in order to increase their robustness towards rf inhomogeneity and enlarge the NMR-responsive sample volume.

Data availability. The experimental NMR data, the simulation data, and the processing and plot scripts for all figures are available at https://doi.org/10.3929/ethz-b-000488476 (Aebischer et al., 2021).

Supplement. The supplement related to this article is available online at: https://doi.org/10.5194/mr-2-523-2021-supplement.

Author contributions. ME designed the research. ZT provided the data about rf field inhomogeneity in probes. KA carried out all measurements and simulations with some help from ME. All the authors discussed and interpreted the results and were involved in writing the paper.

Competing interests. Matthias Ernst is an executive editor of $M R$.

Disclaimer. Publisher's note: Copernicus Publications remains neutral with regard to jurisdictional claims in published maps and institutional affiliations.

Acknowledgements. We would like to thank Perunthiruthy K. Madhu, Kaustubh Mote, and Johannes Hellwagner for the insightful discussions about theory and the experimental implementation of homonuclear decoupling. Beat H. Meier and Alexander Barnes are acknowledged for providing measurement time for the project.
Financial support. This research has been supported by the Schweizerischer Nationalfonds zur Förderung der Wissenschaftlichen Forschung (grant no. 200020_188988) and the Czech Science Foundation (GACR; grant no. 20-00166J).

Review statement. This paper was edited by Bernd Reif and reviewed by Malcolm Levitt and one anonymous referee.

\section{References}

Aebischer, K., Wili, N., Tošner, Z., and Ernst, M.: Using nutationfrequency-selective pulses to reduce radio-frequency field inhomogeneity in solid-state NMR, Magn. Reson., 1, 187-195, https://doi.org/10.5194/mr-1-187-2020, 2020.

Aebischer, K., Tošner, Z., and Ernst, M.: Effects of radial radiofrequency field inhomogeneity on MAS solid-state NMR experiments, ETH Zurich [data set], https://doi.org/10.3929/ethzb-000488476, 2021.

Barnaal, D. and Lowe, I. J.: Effects of Rotating Magnetic Fields on Free-Induction Decay Shapes, Phys. Rev. Lett., 11, 258-260, https://doi.org/10.1103/PhysRevLett.11.258, 1963.

Bielecki, A., Kolbert, A. C., and Levitt, M. H.: Frequency-switched pulse sequences: homonuclear decoupling and dilute spin NMR in solids, Chem. Phys. Lett., 155, 341-346, 1989.

Bielecki, A., Kolbert, A. C., De Groot, H. J. M., Griffin, R. G., and Levitt, M. H.: Frequency-switched Lee-Goldburg sequences in solids, Advances in Magnetic and Optical Resonance, 14, 111124, 1990.

Charmont, P., Lesage, A., Steuernagel, S., Engelke, F., and Emsley, L.: Sample Restriction Using Magnetic Field Gradients in HighResolution Solid-State NMR, J. Magn. Reson., 145, 334-339, https://doi.org/10.1006/jmre.2000.2117, 2000.

Charmont, P., Sakellariou, D., and Emsley, L.: Sample Restriction Using Radiofrequency Field Selective Pulses in HighResolution Solid-State NMR, J. Magn. Reson., 154, 136-141, https://doi.org/10.1006/jmre.2001.2467, 2002.

Cheng, V., Suzukawa, H., and Wolfsberg, M.: Investigations of a nonrandom numerical method for multidimensional integration, J. Chem. Phys., 59, 3992-3999, 1973.

Dyson, F. J.: The Radiation Theories of Tomonaga, Schwinger, and Feynman, Phys. Rev., 75, 486-502, https://doi.org/10.1103/PhysRev.75.486, 1949.

Elbayed, K., Dillmann, B., Raya, J., Piotto, M., and Engelke, F.: Field modulation effects induced by sample spinning: application to high-resolution magic angle spinning NMR, J. Magn. Reson., 174, 2-26, https://doi.org/10.1016/j.jmr.2004.11.017, 2005.

Engelke, F.: Electromagnetic wave compression and radio frequency homogeneity in NMR solenoidal coils: computational approach, Concept. Magnetic Res., 15, 129-155, 2002.

Frantsuzov, I., Vasa, S. K., Ernst, M., Brown, S. P., Zorin, V., Kentgens, A. P. M., and Hodgkinson, P.: Rationalising Heteronuclear Decoupling in Refocussing Applications of Solid-State NMR Spectroscopy, ChemPhysChem, 18, 394-405, https://doi.org/10.1002/cphc.201601003, 2017.

Geen, H. and Freeman, R.: Band-selective radiofrequency pulses, J. Magn. Reson., 93, 93-141, 1991. 
Goldburg, W. I. and Lee, M.: Nuclear Magnetic Resonance Line Narrowing by a Rotating rf Field, Phys. Rev. Lett., 11, 255-258, https://doi.org/10.1103/PhysRevLett.11.255, 1963.

Goldman, M. and Tekely, P.: Effect of radial RF field on MAS spectra, CR Acad. Sci. II C, 4, 795-800, 2001.

Guenneugues, M., Berthault, P., and Desvaux, H.: A Method for Determining $B_{1}$ Field Inhomogeneity. Are the Biases Assumed in Heteronuclear Relaxation Experiments Usually Underestimated?, J. Magn. Reson., 136, 118-126, https://doi.org/10.1006/jmre.1998.1590, 1999.

Gullion, T. and Schaefer, J.: Rotational-echo doubleresonance NMR, J. Magn. Reson., 81, 196-200, https://doi.org/10.1016/0022-2364(89)90280-1, 1989a.

Gullion, T. and Schaefer, J.: Detection of Weak Heteronuclear Dipolar Coupling by Rotational-Echo Double-Resonance Nuclear Magnetic Resonance, Advances in Magnetic and Optical Resonance, 13, 57-83, https://doi.org/10.1016/B978-0-12-0255139.50009-4, 1989b.

Gullion, T., Baker, D. B., and Conradi, M. S.: New, compensated Carr-Purcell sequences, J. Magn. Reson., 89, 479-484, https://doi.org/10.1016/0022-2364(90)90331-3, 1990.

Hartmann, S. R. and Hahn, E. L.: Nuclear double resonance in the rotating frame, Phys. Rev., 128, 2042, https://doi.org/10.1103/PhysRev.128.2042, 1962.

Hediger, S., Meier, B. H., and Ernst, R. R.: Adiabatic passage Hartmann-Hahn cross polarization in NMR under magic angle sample spinning, Chem. Phys. Lett., 240, 449-456, 1995.

Hellwagner, J., Sharma, K., Tan, K. O., Wittmann, J. J., Meier, B. H., Madhu, P. K., and Ernst, M.: Optimizing symmetrybased recoupling sequences in solid-state NMR by pulsetransient compensation and asynchronous implementation, J. Chem. Phys., 146, 244202, https://doi.org/10.1063/1.4989542, 2017.

Hellwagner, J., Grunwald, L., Ochsner, M., Zindel, D., Meier, B. H., and Ernst, M.: Origin of the residual line width under frequencyswitched Lee-Goldburg decoupling in MAS solid-state NMR, Magn. Reson., 1, 13-25, https://doi.org/10.5194/mr-1-13-2020, 2020.

Hohwy, M., Jakobsen, H. J., Eden, M., Levitt, M. H., and Nielsen, N. C.: Broadband dipolar recoupling in the nuclear magnetic resonance of rotating solids: A compensated C7 pulse sequence, J. Chem. Phys., 108, 2686-2694, 1998.

Hong, M.: Oligomeric Structure, Dynamics, and Orientation of Membrane Proteins from Solid-State NMR, Structure, 14, 17311740, https://doi.org/10.1016/j.str.2006.10.002, 2006.

Hoult, D. I. and Richards, R. E.: The signal-to-noise ratio of the nuclear magnetic resonance experiment, J. Magn. Reson., 24, 7185, https://doi.org/10.1016/0022-2364(76)90233-X, 1976.

Idziak, S. and Haeberlen, U.: Design and construction of a high homogeneity rf coil for solid-state multiple-pulse NMR, J. Magn. Reson., 50, 281-288, https://doi.org/10.1016/00222364(82)90058-0, 1982

Jain, M. G., Mote, K. R., Hellwagner, J., Rajalakshmi, G., Ernst, M., Madhu, P. K., and Agarwal, V.: Measuring strong one-bond dipolar couplings using REDOR in magic-angle spinning solid-state NMR, J. Chem. Phys., 150, 134201, https://doi.org/10.1063/1.5088100, 2019.

Jaroniec, C. P., Tounge, B. A., Rienstra, C. M., Herzfeld, J., and Griffin, R. G.: Recoupling of heteronuclear dipolar interactions with rotational-echo double-resonance at high magic-angle spinning frequencies, J. Magn. Reson., 146, 132-139, 2000.

Jia, L., Liang, S., Sackett, K., Xie, L., Ghosh, U., and Weliky, D. P.: REDOR solid-state NMR as a probe of the membrane locations of membrane-associated peptides and proteins, J. Magn. Reson., 253, 154-165, https://doi.org/10.1016/j.jmr.2014.12.020, 2015.

Lee, M. and Goldburg, W. I.: Nuclear-Magnetic-Resonance Line Narrowing by a Rotating rf Field, Phys. Rev., 140, A1261A1271, https://doi.org/10.1103/PhysRev.140.A1261, 1965.

Lee, Y. K., Kurur, N. D., Helmle, M., Johannessen, O. G., Nielsen, N. C., and Levitt, M. H.: Efficient dipolar recoupling in the NMR of rotating solids. A sevenfold symmetric radiofrequency pulse sequence, Chem. Phys. Lett., 242, 304-309, 1995.

Leskes, M., Madhu, P., and Vega, S.: Floquet theory in solid-state nuclear magnetic resonance, Prog. Nucl. Mag. Res. Sp., 57, 345380, https://doi.org/10.1016/j.pnmrs.2010.06.002, 2010.

Levitt, M. H.: Symmetry-Based Pulse Sequences in Magic-Angle Spinning Solid-State NMR, in: eMagRes, edited by: Harris, R. K. and Wasylishen, R. L., John Wiley, Chichester, https://doi.org/10.1002/9780470034590.emrstm0551, 2007.

Levitt, M. H., Oas, T. G., and Griffin, R. G.: Rotary Resonance Recoupling in Heteronuclear Spin Pair Systems, Israel J. Chem., 28, 271-282, 1988.

Li, C., Mo, Y., Hu, J., Chekmenev, E., Tian, C., Gao, F. P., Fu, R., Gor'kov, P., and Brey, W. Cross, T. A.: Analysis of RF heating and sample stability in aligned static solid-state NMR spectroscopy, J. Magn. Reson., 180, 51-57, https://doi.org/10.1016/j.jmr.2006.01.013, 2006.

Lindon, J. C., Beckonert, O. P., Holmes, E., and Nicholson, J. K.: High-resolution magic angle spinning NMR spectroscopy: Application to biomedical studies, Prog. Nucl. Mag. Res. Sp., 2, 79-100, 2009.

Maier, L. C. and Slater, J. C.: Field Strength Measurements in Resonant Cavities, J. Appl. Phys., 23, 68-77, https://doi.org/10.1063/1.1701980, 1952.

Metz, G., Wu, X., and Smith, S.: Ramped-Amplitude Cross Polarization in Magic-Angle-Spinning NMR, J. Magn. Reson. Ser. A, 110, 219-227, https://doi.org/10.1006/jmra.1994.1208, 1994.

Michal, C. A. and Jelinski, L. W.: REDOR 3D: Heteronuclear Distance Measurements in Uniformly Labeled and Natural Abundance Solids, J. Am. Chem. Soc., 119, 9059-9060, https://doi.org/10.1021/ja9711730, 1997.

Mithu, V. S., Tan, K. O., and Madhu, P. K.: Selective inversion of $1 \mathrm{H}$ resonances in solid-state nuclear magnetic resonance: Use of double-DANTE pulse sequence, J. Magn. Reson., 237, 11-16, 2013.

Mote, K. R., Agarwal, V., and Madhu, P. K.: Five decades of homonuclear dipolar decoupling in solid-state NMR: Status and outlook, Prog. Nucl. Mag. Res. Sp., 97, 1-39, 2016.

Nielsen, N. C., Strass $\varnothing$, L. A., and Nielsen, A. B.: Dipolar Recoupling, Springer Berlin Heidelberg, Berlin, Heidelberg, 1-45, https://doi.org/10.1007/128_2011_129, 2012.

Nishimura, K., Fu, R., and Cross, T. A.: The Effect of RF Inhomogeneity on Heteronuclear Dipolar Recoupling in Solid State NMR: Practical Performance of SFAM and REDOR, J. Magn. Reson., 152, 227-233, https://doi.org/10.1006/jmre.2001.2410, 2001.

Odedra, S. and Wimperis, S.: Imaging of the $B_{1}$ distribution and background signal in a MAS NMR probehead using in- 
homogeneous $B_{0}$ and $B_{1}$ fields, J. Magn. Reson., 231, 95-99, https://doi.org/10.1016/j.jmr.2013.04.002, 2013.

Paulson, E. K., Martin, R. W., and Zilm, K. W.: Cross polarization, radio frequency field homogeneity, and circuit balancing in high field solid state NMR probes, J. Magn. Reson., 171, 314-323, https://doi.org/10.1016/j.jmr.2004.09.009, 2004.

Piotto, M., Bourdonneau, M., Furrer, J., Bianco, A., Raya, J., and Elbayed, K.: Destruction of magnetization during TOCSY experiments performed under magic angle spinning: effect of radial B1 inhomogeneities, J. Magn. Reson., 149, 114-118, https://doi.org/10.1006/jmre.2001.2287, 2001

Privalov, A. F., Dvinskikh, S. V., and Vieth, H.-M.: Coil Design for Large-Volume High- $B_{1}$ Homogeneity for Solid-State NMR Applications, J. Magn. Reson. Ser. A, 123, 157-160, https://doi.org/10.1006/jmra.1996.0229, 1996.

Purusottam, R. N., Bodenhausen, G., and Tekely, P.: Effects of inherent rf field inhomogeneity on heteronuclear decoupling in solid-state NMR, Chem. Phys. Lett., 635, 157-162, https://doi.org/10.1016/j.cplett.2015.06.051, 2015.

Rienstra, C. M., Tucker-Kellogg, L., Jaroniec, C. P., Hohwy, M., Reif, B., McMahon, M. T., Tidor, B., Lozano-Pérez, T., and Griffin, R. G.: De novo determination of peptide structure with solid-state magic-angle spinning NMR spectroscopy, P. Natl. Acad. Sci. USA, 99, 10260-10265, https://doi.org/10.1073/pnas.152346599, 2002.

Schanda, P., Meier, B. H., and Ernst, M.: Quantitative analysis of protein backbone dynamics in microcrystalline ubiquitin by solid-state NMR spectroscopy, J. Am. Chem. Soc., 132, 1595715967, 2010.

Schanda, P., Meier, B. H., and Ernst, M.: Accurate measurement of one-bond $\mathrm{H}-\mathrm{X}$ heteronuclear dipolar couplings in MAS solidstate NMR, J. Magn. Reson., 210, 246-259, 2011.

Scholz, I., Hodgkinson, P., Meier, B. H., and Ernst, M.: Understanding two-pulse phase-modulated decoupling in solid-state NMR, J. Chem. Phys., 130, 114510, https://doi.org/10.1063/1.3086936, 2009.

Scholz, I., van Beek, J. D., and Ernst, M.: Operator-based Floquet theory in solid-state NMR, Solid State Nucl. Mag., 37, 39-59, https://doi.org/10.1016/j.ssnmr.2010.04.003, 2010.
Smith, S., Levante, T., Meier, B., and Ernst, R.: Computer simulations in magnetic resonance. An object-oriented programming approach, J. Magn. Reson., 106, 75-105, 1994.

States, D. J., Haberkorn, R. A., and Ruben, D. J.: A twodimensional nuclear Overhauser experiment with pure absorption phase in four quadrants, J. Magn. Reson., 48, 286-292, 1982.

Stejskal, E., Schaefer, J., and Waugh, J.: Magic-angle spinning and polarization transfer in proton-enhanced NMR, J. Magn. Reson., 28, 105-112, 1977.

Tan, K. O., Rajeswari, M., Madhu, P. K., and Ernst, M.: Asynchronous symmetry-based sequences for homonuclear dipolar recoupling in solid-state nuclear magnetic resonance, J. Chem. Phys., 142, 065101, https://doi.org/10.1063/1.4907275, 2015.

Tan, K. O., Agarwal, V., Meier, B. H., and Ernst, M.: A generalized theoretical framework for the description of spin decoupling in solid-state MAS NMR: Offset effect on decoupling performance, J. Chem. Phys., 145, 094201, https://doi.org/10.1063/1.4961909, 2016.

Tekely, P. and Goldman, M.: Radial-field sidebands in MAS, J. Magn. Reson., 148, 135-141, 2001.

Torrey, H. C.: Transient Nutations in Nuclear Magnetic Resonance, Phys. Rev., 76, 1059-1068, https://doi.org/10.1103/PhysRev.76.1059, 1949.

Tošner, Z., Purea, A., Struppe, J. O., Wegner, W., Engelke, F., Glaser, S. J., and Reif, B.: Radiofrequency fields in MAS solid state NMR probes, J. Magn. Reson., 284, 20-32, https://doi.org/10.1016/j.jmr.2017.09.002, 2017.

Tošner, Z., Sarkar, R., Becker-Baldus, J., Glaubitz, C., Wegner, S., Engelke, F., Glaser, S. J., and Reif, B.: Overcoming Volume Selectivity of Dipolar Recoupling in Biological Solid-State NMR Spectroscopy, Angew. Chem. Int. Edit., 57, 14514-14518, https://doi.org/10.1002/anie.201805002, 2018. 